STRUCTURAL SCIENCE CRYSTAL ENGINEERING MATERIALS

ISSN 2052-5206

Received 7 November 2014

Accepted 17 March 2015

Edited by M. de Boissieu, SIMaP, France

Keywords: transmission electron microscopy; powder diffraction; anion-deficient perovskites; modulated structures.

B-IncStrDB references: 10542EJs2b2; 10562EplZqM; 10582EnDDYe; 10602Ed3v26; 10622E902Zs

Supporting information: this article has supporting information at journals.iucr.org/b

\section{Synergy between transmission electron microscopy and powder diffraction: application to modulated structures}

\author{
Dmitry Batuk, $^{\mathrm{a} *}$ Maria Batuk, ${ }^{\mathrm{a}}$ Artem M. Abakumov ${ }^{\mathrm{a}, \mathrm{b}}$ and Joke Hadermann ${ }^{\mathrm{a}}$
}

${ }^{\mathbf{a}}$ EMAT, University of Antwerp, Groenenborgerlaan 171, B-2020 Antwerp, Belgium, and ${ }^{\mathbf{b}}$ Chemistry Department, Moscow State University, 119991 Moscow, Russian Federation. *Correspondence e-mail: dmitry.batuk@uantwerpen.be

The crystal structure solution of modulated compounds is often very challenging, even using the well established methodology of single-crystal Xray crystallography. This task becomes even more difficult for materials that cannot be prepared in a single-crystal form, so that only polycrystalline powders are available. This paper illustrates that the combined application of transmission electron microscopy (TEM) and powder diffraction is a possible solution to the problem. Using examples of anion-deficient perovskites modulated by periodic crystallographic shear planes, it is demonstrated what kind of local structural information can be obtained using various TEM techniques and how this information can be implemented in the crystal structure refinement against the powder diffraction data. The following TEM methods are discussed: electron diffraction (selected area electron diffraction, precession electron diffraction), imaging (conventional high-resolution TEM imaging, highangle annular dark-field and annular bright-field scanning transmission electron microscopy) and state-of-the-art spectroscopic techniques (atomic resolution mapping using energy-dispersive X-ray analysis and electron energy loss spectroscopy).

\section{Introduction}

The emphasis of modern research is greatly shifted towards practical applications, where novel materials play an important role. Understanding structure-property relationships has always been considered as a key component for the discovery and development of materials with new or improved functionalities. The physical properties of the materials might depend on a specific ordering of defects, on subtle and often competing structure distortions and on electronic instabilities, as well as on a delicate interplay between these factors. Owing to tremendous progress in X-ray and neutron diffraction instrumentation and in transmission electron microscopy (TEM), it is now well established that in many functional materials these factors result in structure modulations. If the modulation periodicity is an irrational multiple of the underlying basic structure period, the structure is incommensurately modulated and aperiodic (i.e. lacks a three-dimensional translational symmetry). There are several origins of incommensurability in the modulated structures. The interplay between interatomic interactions and lattice strain enforces point defects to distribute as homogeneously as possible and order into modulated uniform patterns. Competing distortion modes (e.g. cooperative deformation of a framework of rigid units, displacements of cations with lone electron pairs, offcentre polar displacements etc.) may result in a modulated frustrated structure. Electronic instabilities and orbital 
ordering can give rise to incommensurate charge and spin density waves. Finally, incommensurability is intrinsic in composite structures, which consist of continuous interpenetrating sublattices with different mutually incommensurate periodicities along certain crystallographic direction(s).

Retrieving detailed information on the modulated structures is still far from trivial and requires crystallographic approaches in $(3+d)$-dimensional space, where $d$ is the number of modulation vectors that depend on the symmetry and the driving force of the modulation. In our brief introduction we will consider a $d=1$ case, as it comprises the overwhelming majority of modulated structures. The reciprocal space of a modulated structure can be described with a diffraction vector $\mathbf{H}=h \mathbf{a}^{*}+k \mathbf{b}^{*}+l \mathbf{c}^{*}+m \mathbf{q}, \quad$ with $\mathbf{q}=\alpha \mathbf{a}^{*}+\beta \mathbf{b}^{*}+\gamma \mathbf{c}^{*}$, where $\mathbf{q}$ is a modulation vector that has $\alpha, \beta, \gamma$ components with respect to the reciprocal lattice basis $\mathbf{a}^{*}, \mathbf{b}^{*}, \mathbf{c}^{*}$ of the parent average structure. The main reflections of the average structure correspond to $m=0$, while the satellite reflections due to the modulation are indexed with integer $m \neq 0$. If at least one of the coefficients $\alpha, \beta$ or $\gamma$ is an irrational number, there will be no common three-dimensional reciprocal lattice fitting the position of the main reflections and the satellites (Fig. 1a). The regular reciprocal lattice of the incommensurately modulated crystal can be restored by considering the structure in a space of higher dimensionality. For a modulation with a single q-vector, the $(3+1)$-dimensional reciprocal space is spanned by the three basis vectors
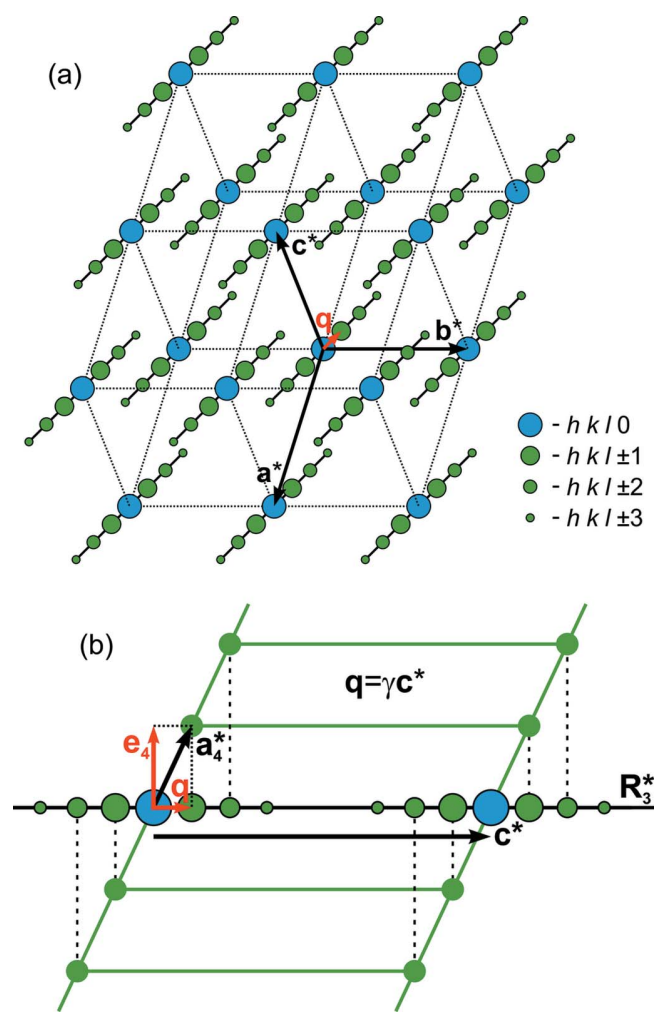

Figure 1

(a) A three-dimensional reciprocal space and $(b)$ a $(3+1)$-dimensional reciprocal lattice of an incommensurately modulated crystal. The main reflections and satellites are marked in blue and green, respectively. $\mathbf{a}_{1}^{*}=\left(\mathbf{a}^{*}, 0\right), \mathbf{a}_{2}^{*}=\left(\mathbf{b}^{*}, 0\right), \mathbf{a}_{3}^{*}=\left(\mathbf{c}^{*}, 0\right)$ and the fourth vector $\mathbf{a}_{4}^{*}=(\mathbf{q}, 0)+\mathbf{e}_{4}$, where $\mathbf{e}_{4}$ is a unit vector, corresponding to the fourth axis orthogonal to the three-dimensional reciprocal space $\boldsymbol{R}_{3}^{*}$ (Fig. $1 b$ ). As a result, the diffraction pattern is a projection of the $(3+1)$-dimensional reciprocal lattice onto the three-dimensional reciprocal space along the $\mathbf{e}_{4}$ direction. Since the diffraction pattern of the modulated structure is described with the $(3+1)$-dimensional reciprocal lattice, the atomic arrangement can be defined in a periodic manner in the $(3+1)$-dimensional direct space. The atomic positions in the superspace are associated with a periodic curve whose shape is defined by the modulation function $\mathbf{U}\left(\bar{x}_{4}\right)$. The three-dimensional structure is recovered by the section of the $(3+1)$ dimensional space with a hyperplane $\boldsymbol{R}_{3}$. In the threedimensional physical space atomic positions in the modulated structure are given by $\mathbf{r}=\mathbf{r}_{0}+\mathbf{n}+\mathbf{U}\left(\mathbf{q} \cdot\left(\mathbf{r}_{0}+\mathbf{n}\right)+t\right)$, where $\mathbf{r}_{0}+\mathbf{n}$ is the position of the atom in the unit cell of the average structure related to the position in the first unit cell $\mathbf{r}_{0}$ by translations $\mathbf{n}, \mathbf{U}$ is the modulation function with the argument $\bar{x}_{4}=\mathbf{q} \cdot\left(\mathbf{r}_{0}+\mathbf{n}\right)+t, t$ is the initial phase of the modulation wave. In the most general case the displacive modulation function can be represented as a Fourier series

$$
\mathbf{U}\left(\bar{x}_{4}\right)=\sum_{n}\left\{\mathbf{A}_{n} \sin \left(2 \pi n \bar{x}_{4}\right)+\mathbf{B}_{n} \cos \left(2 \pi n \bar{x}_{4}\right)\right\}
$$

where $\mathbf{A}_{n}=\left(A_{n, x}, A_{n, y}, A_{n, z}\right), \mathbf{B}_{n}=\left(B_{n, x}, B_{n, y}, B_{n, z}\right)$ are Fourier coefficients for the sine and cosine terms, respectively, $n$ is the order of the Fourier term. The modulation functions can be applied to different quantities such as occupancy factors, atomic displacement parameters and magnetic moment vectors, thus giving a comprehensive description of the modulated structure. Further details on multi-dimensional crystallography can be found in the seminal articles, books and recent reviews (de Wolff, 1974, 1977; Janner \& Janssen, 1977; van Smaalen, 2004, 2007; Janssen et al., 2006, 2007; Wagner \& Schönleber, 2009; Janssen \& Janner, 2014).

Solution of a modulated structure implies not only finding the atomic positions in the unit cell of the average structure, but also determining the shape of the modulation functions. The structure solution becomes more challenging if only powder diffraction data are available. Even for conventional non-modulated structures, a round robin test has demonstrated that solving the structure using powder diffraction data is still considerably more difficult than tackling the same problem with single-crystal diffraction (Le Bail et al., 2009). An incommensurate modulation adds an extra degree of complexity. Most difficulties are associated with the fact that the powder diffraction pattern is a one-dimensional projection of the reciprocal lattice. For the incommensurately modulated structures this means that the hklm reflections on powder patterns form a very dense set. If $p$ is the number of the main reflections $(m=0)$, the total number of reflections is $(2 m+1) p$, which includes the main reflections and the satellites up to the order $m$. Yet, profile decomposition and Rietveld refinement are still possible because in most cases the intensities of the satellite reflections rapidly decrease with their increasing order (i.e. with increasing $|m|$ ). This allows considering the set 
of reflections as finite and discrete. On the other hand, frequent overlap of weak satellites with intense main reflections and a generally low signal-to-noise ratio strongly hinder accurate determination of the positions and intensities of the satellite reflections. Overlap of the main reflections and satellites may also impede application of the commonly used approach of the two-stage structure refinement, where first the average structure is refined using the main reflections only and then the parameters of the modulation functions are introduced into the refinement.

To solve a modulated structure, one should take the following steps:

(1) identify and index the main reflections, determine the lattice parameters of the parent structure;

(2) identify the satellite reflections assuming the corresponding q-vector(s);

(3) index the satellites and find the components $\alpha, \beta, \gamma$ of the q-vector(s);

(4) determine the crystal system and lattice centring compatible with the irrational and rational components of the q-vector(s);

(5) determine the reflection conditions and superspace group;

(6) construct a structure model in superspace $\equiv$ solve the structure;

(7) refine the structure: atomic coordinates, occupancy factors, atomic displacement parameters and parameters of the modulation functions;

(8) validate the structure solution in three-dimensional space.

More than 100 modulated structures have been refined so far from powder diffraction data. We have briefly analysed the common practice in this field using a set of 106 structures reported in the period of 1989-2014 (see the list of references in the supporting information, Table S1). It might not be comprehensive, but sufficiently representative to pick up the trends. Laboratory X-ray powder diffraction (XPD), highresolution synchrotron X-ray powder diffraction (SXPD) and neutron powder diffraction (NPD) are widely employed for the analysis of modulated structures. Nearly one third of the structures were refined using the Rietveld method solely from laboratory XPD. In spite of its obvious disadvantages (medium resolution, low signal-to-noise ratio, difficulties with locating the 'light' atoms), this approach remains adequate for the analysis of modulated structures, although it gradually becomes replaced with the more advanced powder diffraction techniques. For materials where the average structure is only weakly perturbed by displacive modulations, laboratory XPD generally allows reliable detection of only first-order satellite reflections and the refinement of harmonic amplitudes up to second order (Dušek et al., 2001; Graetsch, 2003, 2007; Righi et al., 2006, 2007, 2008; Larsson, García-García et al., 2007). Measuring the diffraction patterns with synchrotron X-ray radiation provides much better visibility of higher-order satellite reflections, e.g. compare Righi et al. (2006) and Singh et al. (2014). High-order satellites are required for the refinement of some composite structures (Onoda et al., 1993;
Isobe et al., 2010; Schmid \& Fung, 2012), however, in the majority of cases the composite powder patterns can be adequately fitted by taking into account satellites of up to second order. For commensurately modulated structures modeled in superspace using discontinuous occupancy modulation functions the maximum order of the satellites should be sufficient to index all reflections of the corresponding supercell structure (Boullay et al., 2003; Morozov et al., 2010), but the highest observable satellite order can be lower for powder diffraction (Boullay et al., 2003).

In more than $95 \%$ of the structures, the refinement from powder data starts with an average structure and/or superspace model, which are either taken from the preceeding single-crystal structure solution in superspace, belong to a commonly known structure type (such as perovskite, $\alpha-\mathrm{PbO}$, scheelite etc.) or derived from previously determined commensurate analogues. To the best of our knowledge, structure solution in superspace from powder diffraction data using direct methods (Hao et al., 1987) or the charge flipping algorithm (Palatinus, 2004) has not been performed so far, although both methods are successfully applied to the powder diffraction patterns of conventional periodic structures (Altomare et al., 2004; Baerlocher et al., 2007). The amplitudes of the displacive and occupational harmonic modulation functions are generally obtained in the refinement starting from arbitrary small values (Yamamoto, 1996; Dušek et al., 2001) and verified against the Fourier maps. In the structures modeled with a set of the step-like occupational modulation functions, the position and width of the atomic domains in superspace are frequently determined through a trial-anderror approach. Refinement of the modulation of atomic displacement (thermal) parameters is, perhaps, beyond the possibilites of powder diffraction techniques, but in some cases it can provide a noticeable improvement of the Rietveld fit (Larsson, García-García et al., 2007).

It appears that the solution of a modulated structure from powder diffraction data is often coupled with a transmission electron microscopy investigation. In the vast majority of cases the role of TEM is auxilliary, yet very important. The application of TEM is mostly confined to selected area electron diffraction (SAED) for studying the geometry of reciprocal space and high-resolution TEM (HRTEM) imaging for confirming the results of the structure refinement.

SAED is particularly useful when combined with powder diffraction techniques: SAED patterns along main crystallographic directions can be obtained from small individual single crystals of the material that constitute the powder sample providing a clear visualization of the reciprocal lattice sections. This enables the detection of satellites, finding the components of the modulation vector(s), identifying reflection sets of composite subsystems and analysing the superspace symmetry. SAED alone has been frequently used for qualitative analysis of the modulated structures (Buseck \& Cowley, 1983; Van Landuyt et al., 1985; Withers, 1989; Withers et al., 2004). The knowledge obtained from SAED greatly facilitates further indexing and interpretation of the powder diffraction patterns (Milne et al., 1985; Onoda et al., 1988; Baldinozzi et 
al., 1996; Boullay, Grebille et al., 1999; Boullay, Hervieu et al., 1999, and other references in the supporting information).

The in-zone SAED patterns reveal the geometry of the reciprocal lattice of the modulated structure. However, the intensity of the reflections is considerably distorted by dynamical effects and multiple scattering, which arise from the strong interaction of electrons with the matter. In fact, the intensity transfer from basic reflections to satellites due to multiple diffraction can strongly enhance the intensities of the latter. It makes the detection of satellites easier, but at the same time artificially increases the maximum order of observable satellite reflections (Steeds et al., 1985; Bird \& Withers, 1986). Besides, it can complicate the analysis of the reflection conditions and the assignment of a superspace group. In some cases dynamical electron diffraction, even when treated in a kinematic approximation, can still provide a rough structure model (Hao et al., 1987; Xiang et al., 1990; Mo et al., 1992). However, the dynamical effects result in large discrepancies between the observed and the calculated structure factors, which can be minimized using the procedure proposed by Sha et al. (1993). It is based on the notion that if the structure model is available, the crystal thickness can be estimated by comparing the experimental diffraction intensities with those obtained by multislice simulations. Then, the observed intensities can be calibrated to produce a set of pseudo-kinematical structure factors that can be used for the structure refinement.

The effect of dynamical scattering can be diminished by changing the diffraction data collection procedure. Vincent \& Midgley (1994) suggested that a reduction in the number of simultaneously excited diffraction beams can drastically suppress the dynamical effects. In the conventional SAED mode the incident electron beam is parallel to the optical axis of the microscope as well as to the zone axis of the crystal. This way an axially symmetrical pattern is produced with many simultaneously excited diffracted beams. Tilting the electron beam away from the optical axis makes the Ewald sphere intersect the reciprocal lattice layer. This produces an arc of reflections with a much smaller number of simultaneously excited diffracted beams. Precession of the beam around the optical axis along a cone causes the arc of reflections to rotate. High enough precession frequency during the acquisition produces an axially symmetric precession electron diffraction (PED) pattern, but with the intensities recorded in quasikinematical conditions. By registering PED patterns along different zone axes, a three-dimensional diffraction data set can be constructed and used for the solution and refinement of the crystal structures (Boulahya et al., 2007; Gemmi \& Nicolopoulos, 2007; Eggeman \& Midgley, 2012; Hadermann et al., 2012; Klein, 2013). However, comparison of incommensurately modulated structures refined from PED and SXPD data shows that the intensities of the satellite reflections in PED are still overestimated due to residual dynamical effects. For example, analysis of the compositional modulation in the $\mathrm{CaEu}_{2} \square\left(\mathrm{WO}_{4}\right)_{4}(\square$-cation vacancy) scheelite using PED data revealed a complete ordering of $\mathrm{Ca}, \mathrm{Eu}$ and cation vacancies described by step-like occupational modulation functions. The refinement of the coefficients of the displacive modulation functions provided excessively large and meaningless atomic displacements (Morozov et al., 2013). Later refinement of this structure from SXPD data demonstrated that the ordering of $\mathrm{Ca}, \mathrm{Eu}$ and cation vacancies is actually incomplete and is better fitted with a harmonic modulation (Abakumov et al., 2014).

The PED data acquisition can be combined with reciprocal space electron tomography. In this mode, a large number of the PED patterns are collected on a crystal being rotated with small angular steps, typically $\sim 1^{\circ}$. The in-zone axis orientations are generally avoided because they are most affected by dynamic diffraction effects (Gorelik et al., 2011; Kolb et al., 2011). This results in three-dimensional electron diffraction data with a high coverage of the reciprocal space, which can be used for the solution of a modulated structure employing the charge flipping algorithm in superspace (Palatinus, 2004; Palatinus et al., 2011; Boullay et al., 2013). The obtained distribution of the scattering density can subsequently be used for introducing discontinuous occupancy modulations and building a model for the Rietveld refinement from XPD data (Boullay et al., 2013).

TEM imaging offers a unique opportunity for visualization of the atomic arrangement of the structure in direct space. Historically, the first imaging mode where atomic resolution was possible was HRTEM. In this mode, the sample is irradiated with a parallel plane-wave electron beam. The elastically scattered electrons that pass through the sample create an interference pattern, which is recorded as a HRTEM image (Williams \& Carter, 2009). HRTEM images were traditionally used to retrieve qualitative direct space information on the type and nature of the modulation (Steeds et al., 1985; Van Landuyt et al., 1985; Matsui et al., 1988; Van Tendeloo et al., 2009). There are few examples where the occupational and/or displacive modulation waves in the layered cuprates were visualized with the HRTEM images using image deconvolution coupled with a so-called phase extension procedure (Fu et al., 1994; Liu et al., 1998). The structure factors were extracted from the Fourier transform of the HRTEM images, which was corrected using the phase-contrast transfer function of the microscope, employing empirically estimated defocus values (for example, with the maximum entropy method) and other known parameters of the microscope (Li, 1997, 2010; Fan, 1999). The obtained set of structure-factor phases of low and medium spatial resolution can then be combined with the experimental reflection intensities from SAED patterns. This generally provides higher spatial resolution, allowing extension of the phases through the Sayre equation modified for the multi-dimensional case. Then the projected crystal potential can be reconstructed using Fourier transforms. Also, HRTEM images play an important role in validation of the modulated structure after the Rietveld refinement. Using a commensurate approximant or just a part of the incommensurately modulated structure of an appropriate size, the theoretical HRTEM images can be computed for different defocus and crystal thickness values using the multislice method. The qualitative correspondence between experimental and calculated images (usually estimated visually) provides a confir- 
mation of the refined structure model (Abakumov et al., 2003; Gillie et al., 2004; Morozov, Arakcheeva et al., 2006; Rusakov et al., 2011).

Transmission electron microscopy and powder diffraction make a perfect marriage being complementary techniques for the investigation of complex structures (McCusker \& Baerlocher, 2009, 2013). However, the field of aperiodic powder crystallography apparently lacks the full synergy of both methods. Abilities of the electron diffraction and imaging techniques for retrieving information on modulated structures are still far from being completely exploited, although few important steps have already been made (particularly in the field of quantitative electron diffraction). In the past two decades the TEM demonstrated tremendous progress, related to the invention and development of aberration-corrected electron optics and scanning transmission electron microscopy methods coupled with spectroscopic techniques such as energy-dispersive X-ray analysis and electron energy loss spectroscopy (Muller, 2009; Van Tendeloo et al., 2012). In this contribution we would like to illustrate these emerging opportunities for the investigation of incommensurately modulated structures of inorganic solids.

As an example, we will use one specific family of oxide structures - anion-deficient perovskites modulated by crystallographic shear (CS) planes, where the anion deficiency is accommodated by periodic non-conservative translational interfaces, somewhat similar to CS planes in $\mathrm{ReO}_{3}$-type oxides (Magnéli et al., 1948; Wadsley, 1955; Tilley, 1970, 1995; Anderson, 1972; Bursill \& Hyde, 1972; Anderson \& Tilley, 1974). These interfaces are typically confined to the $(h 0 l)_{\mathrm{p}}$ planes with the displacement (shear) vector $\mathbf{R}_{0}=1 / 2[110]_{\mathrm{p}}$, where the subscript 'p' denotes the parent perovskite $A B \mathrm{O}_{3}$ structure (Bougerol et al., 2002; Abakumov et al., 2006; Abakumov, Hadermann et al., 2008). As a result of this shear operation, the corner-sharing $\mathrm{BO}_{6}$ octahedra of the perovskite structure along the CS planes are transformed into edgesharing $\mathrm{BO}_{5}$ distorted tetragonal pyramids, hence reducing the oxygen content (see further in the text). The specific atomic configuration along the interfaces is stabilized by the lone electron pair $A$ cations $\left(\mathrm{Pb}^{2+}, \mathrm{Bi}^{3+}\right)$ due to their ability to adopt incomplete and asymmetric oxygen coordination. The strain associated with the atomic rearrangement at the interfaces is mitigated through a substantial relaxation displacement $\mathbf{R}_{1} \simeq \delta[001](\delta=1 / 3-1 / 2)$, which does not change the connectivity of the metal-oxygen polyhedra, but restores normal interatomic distances at the interfaces, at the same time causing incommensurability. The structure solution of these materials posed a challenge that could not be tackled using solely powder diffraction methods, without the help of TEM.

\section{Experimental}

Anion-deficient perovskites $(\mathrm{Pb}, \mathrm{Bi})_{1-x} \mathrm{Fe}_{1+x} \mathrm{O}_{3-y}$ were synthesized using solid-state reaction from corresponding binary oxides $\mathrm{PbO}$ (Sigma-Aldrich, $>99.9 \%$ ), $\mathrm{Bi}_{2} \mathrm{O}_{3}$ (Aldrich, $99.9 \%), \mathrm{Fe}_{2} \mathrm{O}_{3}$ (Sigma-Aldrich, > 99\%). The reaction was conducted in several annealing steps, the conditions of which were determined empirically and can be found in an earlier report (Abakumov et al., 2011). Five compounds of the $(\mathrm{Pb}, \mathrm{Bi})_{1-x} \mathrm{Fe}_{1+x} \mathrm{O}_{3-y}$ series with $\mathrm{Bi}$ concentrations of 10,20 , 30,44 and 56 at.\% in the $A$ cation sublattice were synthetized in an amount of $10 \mathrm{~g}$ each for neutron powder diffraction experiments.

X-ray powder diffraction data were used to monitor the synthesis progress and the phase composition. XPD patterns were collected on a Huber G670 Guinier diffractometer $\left(\mathrm{Cu} K \alpha_{1}\right.$ radiation, curved $\mathrm{Ge}$ monochromator, transmission mode, image plate). Neutron powder diffraction data were collected with the high-resolution powder diffractometer (HRPT) at the Laboratory for Neutron Scattering and Imaging (LNS) at the Paul Scherrer Institut (PSI), Switzerland (Fischer et al., 2000). Crystal structure refinements were performed using the JANA2006 program (Petrríček et al., 2014).

Samples for transmission electron microscopy were made by grinding powders in ethanol and depositing a few drops of dispersions on holey carbon grids. The electron diffraction patterns were taken on an FEI Tecnai G2 microscope operated at $200 \mathrm{kV}$. High-angle annular dark-field scanning transmission electron microscopy (HAADF-STEM) and annular bright-field scanning transmission electron microscopy (ABFSTEM) images were recorded with a probe aberrationcorrected FEI Titan 'cubed' microscope at $300 \mathrm{kV}$. Electron energy loss spectroscopy (EELS) was performed with the same microscope operated at $120 \mathrm{kV}$ in monochromated STEM mode (convergence semi-angle $18.5 \mathrm{mrad}$, monochromator excited to provide an energy resolution of $\sim 250 \mathrm{meV}$, beam current $\sim 60 \mathrm{pA}$, probe size $\sim 1.5 \AA$, inner collection semi-angle and spectrometer acceptance semi-angle $160 \mathrm{mrad}$ ). Atomic resolution energy-dispersive X-ray (EDX) elemental mapping was carried out with an FEI Titan 'cubed' microscope equipped with a Super-X detector and operated at $200 \mathrm{kV}$. Simulated STEM images were calculated using QSTEM software (Koch, 2002).

\section{Reciprocal space}

Perovskites with the CS planes belong to the interfacemodulated structures, where the parent perovskite structure undergoes abrupt changes at the interfaces. These periodic changes can be modelled in superspace using discontinuous occupational modulations with the finite size of atomic domains, similar to quasicrystals (Michiue et al., 2005, 2006, 2007; Bolotina, 2007; Izaola et al., 2007). The diffraction patterns of such structures consist of linear arrays of reflections centred at the reciprocal lattice nodes of the parent structure. Part of the main reflections can be extinct because of the shift of the parent structure blocks at the interfaces. The satellite reflections can be as strong as the main reflections because of discontinuous occupancy modulations and associated strong atomic displacements due to structure relaxation at the interfaces (Van Landuyt et al., 1970, 1985; Van Dyck et al., 1987). For powder diffraction, this makes the differentia- 
tion between the main and satellite reflections exceptionally difficult, if ever possible. In other words, it hinders the indexing of the XPD patterns and the determination of the lattice parameters of the parent structure and the components of the modulation vector, which are the essential prerequisites for embedding the modulated structure into higher-dimensional space and constructing a superspace model for Rietveld refinement. In this case, the reciprocal space can be overviewed from a series of the reciprocal lattice sections observed as SAED patterns. For the $(\mathrm{Pb}, \mathrm{Bi})_{1-x} \mathrm{Fe}_{1+x} \mathrm{O}_{3-y}$ family of CS structures (Abakumov et al., 2011) linking the in-zone SAED with XPD was the only way to index the XPD patterns. The correspondence between SAED and XPD patterns becomes apparent if the reflection positions from the SAED pattern are projected onto the peaks in the XPD pattern plotted as a function of inverse interplanar spacing 1/d (Fig. 2). The linking can be accomplished by comparing the interplanar

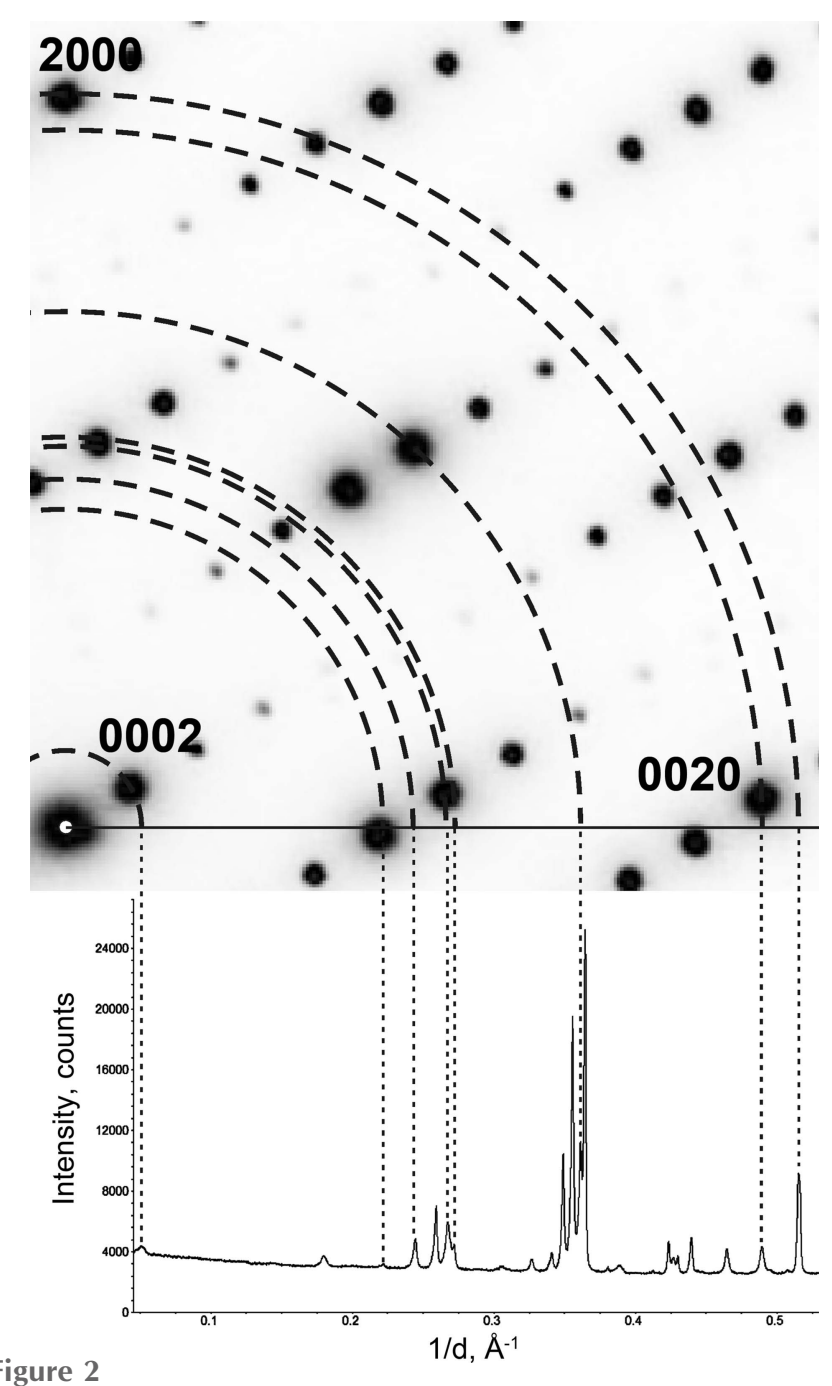

Figure 2

Projecting an electron diffraction pattern on a powder X-ray diffraction pattern. Note that not all reflections on the powder pattern are present on the electron diffraction pattern. These reflections belong to other reciprocal lattice sections. Electron diffraction intensities do not match well the X-ray diffraction intensities because the former are dynamically distorted and the latter are affected by the reflection overlap. distances, $d$, for the reflections on the SAED and XPD patterns. However, one should keep in mind that the accuracy of the interplannar distance determination from SAED patterns is somewhat two-three orders of magnitude lower than that from XPD data. Besides, extreme care should be taken while comparing reflection intensities in the SAED and XPD patterns. The SAED pattern represents a section of the reciprocal lattice of the crystal, where the intensities are distorted due to dynamical effects and multiple scattering as well as possible misorientation of the crystal. The XPD pattern represents a projection of the three-dimensional reciprocal lattice into a one-dimensional dataset, so that the intensity of the peak depends on a multiplicity factor of the corresponding reflection and possible accidental overlaps (Pecharsky \& Zavalij, 2009). Nevertheless, in most cases for a given row of reflections (main reflection + satellites) the intensity of the main reflection is higher (if not extinct by the symmetry) and the intensity of satellites decreases with increasing order. Thus, selecting the reciprocal lattice vectors of the parent structure and associated arrays of satellites can be performed following the requirement to maintain the highest intensity for low-order satellite reflections (Fig. 3). After indexing the SAED patterns and establishing the correspondence between SAED and XPD, the hklm indexes can be assigned to all the reflections in the XPD pattern. Then the unit-cell parameters of the parent structure and the components of the modulation vector can be calculated using a general expression for the diffraction vector $\mathbf{H}=(h+m \alpha) \mathbf{a}^{*}+(k+m \beta) \mathbf{b}^{*}+$ $(l+m \gamma) \mathbf{c}^{*}$. Finally, the unit-cell parameters and modulation vector components can be refined using full profile decomposition, e.g. with the Le Bail fit (Fig. 3).

Following this procedure, the XPD data for all compounds of the $(\mathrm{Pb}, \mathrm{Bi})_{1-x} \mathrm{Fe}_{1+x} \mathrm{O}_{3-y}$ series can be indexed with a monoclinic perovskite basic cell with $a_{\mathrm{p}} \simeq b_{\mathrm{p}} \simeq c_{\mathrm{p}} \simeq 3.9 \AA$ close to $90^{\circ}$ and the modulation vector $\mathbf{q}=\alpha \mathbf{a}^{*}+\gamma \mathbf{c}^{*}$ having two irrational components $\alpha$ and $\gamma$. The systematic reflection condition hklm: $h+k+l+m=2 n$ observed from both SAED and XPD patterns suggests the $(3+1)$-dimensional space group $X 2 / m(\alpha 0 \gamma) 00$ with the centring vector $X=$ $[1 / 2,1 / 2,1 / 2,1 / 2]$, which is a nonstandard setting of the $B 2 / m(\alpha \beta 0) 00$ superspace group (Stokes et al., 2011). The SAED patterns provided a clear overview of the compositionally related variations of the modulation vector. Fig. 4 (upper row) demonstrates the SAED patterns of $(\mathrm{Pb}, \mathrm{Bi})_{1-x} \mathrm{Fe}_{1+x} \mathrm{O}_{3-y}$ together with the corresponding direct space images (bottom row). As the $\mathrm{Bi}^{3+}$ content increases, the orientation of the satellite reflections remains practically the same, while the spacing between the satellite reflections (i.e. the length of the modulation vector) decreases. In direct space it implies that the orientation of the CS planes with respect to the perovskite sublattice stays practically the same being close to the $(509)_{\mathrm{p}}$ perovskite lattice plane, but the thickness of the perovskite blocks (structure fragment between the planes) increases (Fig. 4, bottom row).

Operating with SAED patterns taken along the most prominent zone axes might not be sufficient for a complete reconstruction of the reciprocal space of the modulated 
structure. The modulation vector can be arbitrarily oriented with respect to the reciprocal lattice axes of the main structure and the satellite reflections can be positioned arbitrarily close to each other. One can easily imagine a situation when for a structure with all three irrational components $(\alpha, \beta, \gamma)$ there will be no reciprocal lattice sections simultaneously containing the modulation vector and more than one row of the main reflections, thus making it difficult to find the relationship between $\mathbf{a}^{*}, \mathbf{b}^{*}, \mathbf{c}^{*}$ and the $\mathbf{q}$ vector. Moreover, if the goal of a diffraction experiment is collecting diffracted intensities, recording only the in-zone SAED patterns might substantially limit the number of measured satellite reflections. Thus, threedimensional reconstruction of the reciprocal space with high spatial resolution of the acquired data is very important for the modulated structures. In the rotation electron diffraction method, mechanical rotation of the crystal is combined with a fine electron beam tilt of $\sim 0.05^{\circ}$ (Zhang et al., 2010). This fills the gap between the subsequent orientations of the crystal and ensures complete coverage of the scanned wedge of the reciprocal space. It results in a very high resolution of the threedimensional reconstructions, as illustrated by Movie 1 in the supporting information that shows an animated recipro- cal space reconstruction for a compound of the $(\mathrm{Pb}, \mathrm{Bi})_{1-x} \mathrm{Fe}_{1+x} \mathrm{O}_{3-y}$ series with 30 at. \% Bi.

\section{Direct space}

The development of high-resolution scanning transmission electron microscopy (STEM) methods opened up new possibilities for direct space visualization of the atomic arrangement in crystalline solids (Nellist, 2011). In the STEM mode the incident electron beam is focused into a very fine probe of $\sim 1 \AA$ wide. The probe scans over the specimen in a raster pattern. Transmitted electrons are collected with an annular detector located in the diffraction plane and the registered intensity is plotted against the position of the probe (Fig. 5). The inner and outer collection angles of the detector define the angular range, in which the scattered electrons are recorded. These angles can be easily changed by changing the excitation of the projection system of the microscope; however, the ratio between the inner and the outer angle is largely defined by the physical parameters of the detector and design of the column. The most commonly used STEM mode is the high-angle annular dark-field STEM (HAADF-STEM).
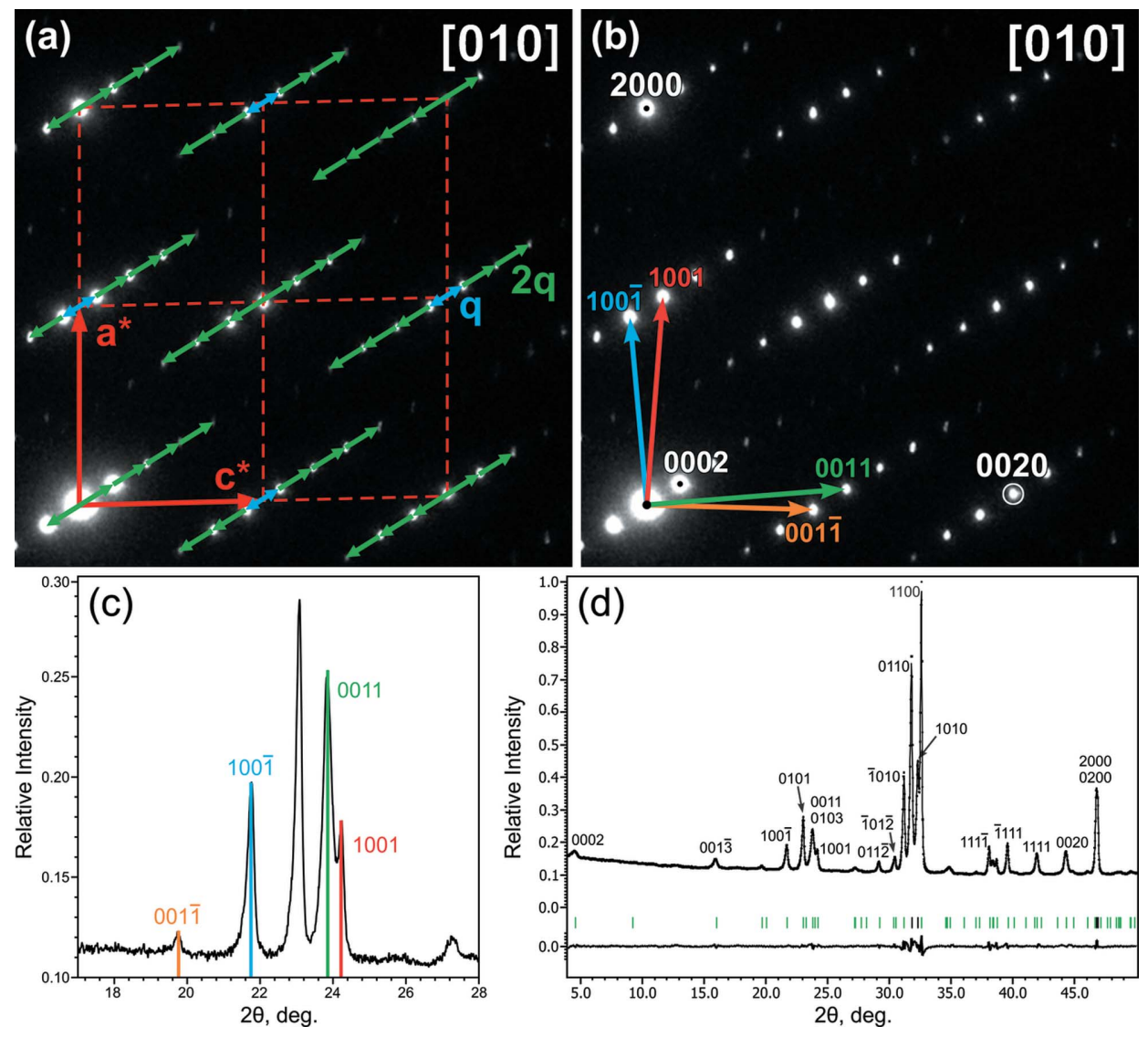

Figure 3

Combining SAED and XPD patterns for determining the crystallographic parameters of the $(\mathrm{Pb}, \mathrm{Bi})_{1-x} \mathrm{Fe}_{1+x} \mathrm{O}_{3-y}$ modulated structures: $(a)$ defining the basic reciprocal lattice vectors $\mathbf{a}^{*}$ and $\mathbf{c}^{*}$ (red) and positioning the satellite arrays (blue arrows mark the separation $|\mathbf{q}|$, green arrows mark the separation $2|\mathbf{q}|$ due to centring in $(3+1)$-dimensional space); (b) reciprocal lattice vectors of the satellite reflections with assigned $\mathrm{hklm}$ indexes; $(c)$ the same satellite reflection identified on the XPD pattern; $(d)$ Le Bail fit showing a complete indexing of the XPD pattern.
In this mode, the detector collects electrons scattered to very high angles. Typically the inner collection angle of the detector is somewhat three times larger than the convergence angle of the probe. This way, the contribution of the Bragg scattering (i.e. coherent elastic scattering on the electrostatic potential of the crystal as whole) is minimized. The HAADFSTEM signal is dominated by the Rutherford scattering (i.e. incoherent elastic electron scattering on the nuclei) and the inelastic thermal diffuse scattering. As a result, the HAADF-STEM signal strongly depends on the chemical composition. For the in-zone axis atomic resolution HAADF-STEM images, the intensity of the atomic columns scales as $Z^{n}$, where $Z$ is the average atomic number of the projected column and $1.6<n<1.9$ (Hartel et al., 1996). For many structures, the interpretation of the HAADF-STEM images is straightforward, because the atomic columns appear as bright dots on a black background and their positions directly correlate with the projected electrostatic potential of the crystal. The contrast is very robust to the defocus and crystal thickness 

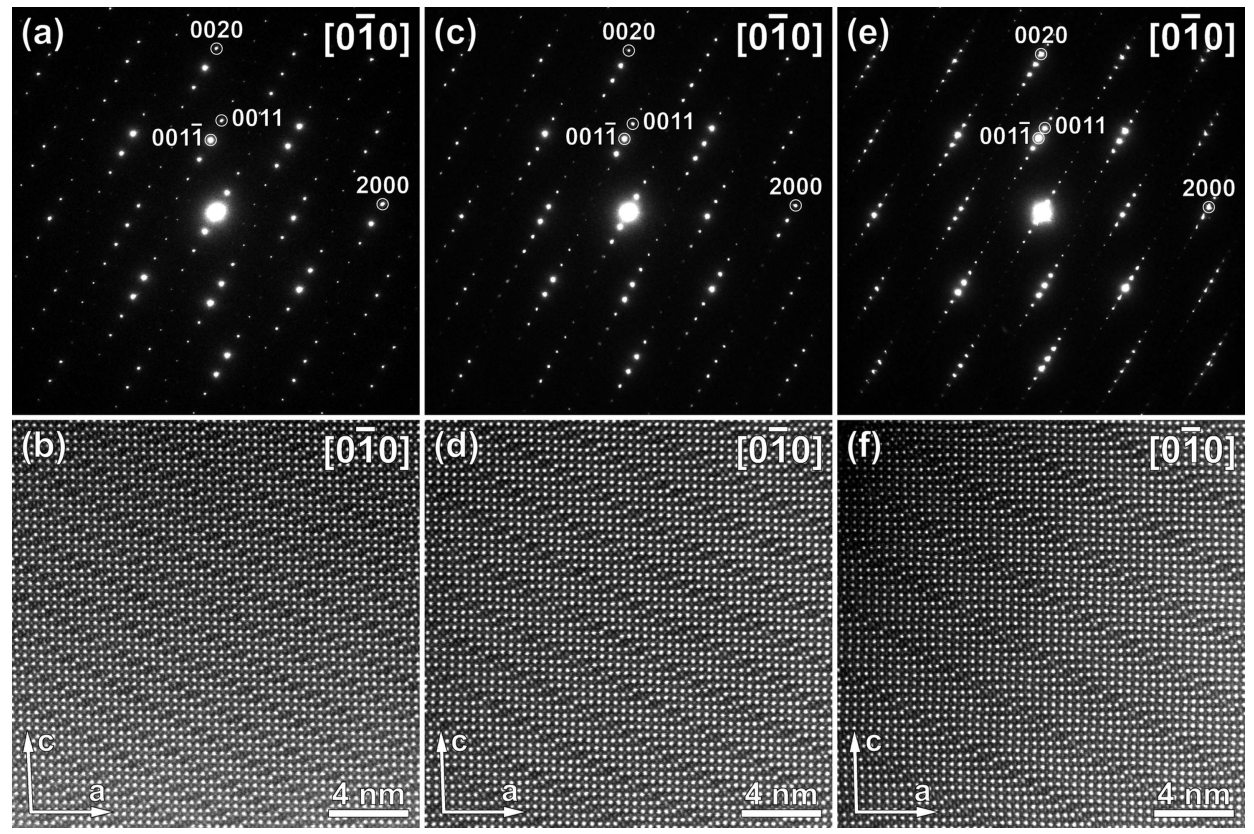

Figure 4

SAED patterns (upper row) and the corresponding HAADF-STEM images (lower row) for three members of the $(\mathrm{Pb}, \mathrm{Bi})_{1-x} \mathrm{Fe}_{1+x} \mathrm{O}_{3-y}$ family with different concentration of $\mathrm{Bi}$ (i.e. different degree of anion deficiency that depends on the $\mathrm{Pb}^{2+} / \mathrm{Bi}^{3+}$ ratio): $(a, b) 10$ at. $\%,(c, d) 30$ at. $\%(e, f) 56$ at. $\%$ of $\mathrm{Bi}$ in the $A$ cation sublattice.

variations and practically insensitive to dynamical effects. In the application to modulated structures the HAADF-STEM images can provide information on the occupational and positional modulation waves, especially if the modulations arise from the ordering of atomic species with sufficiently large

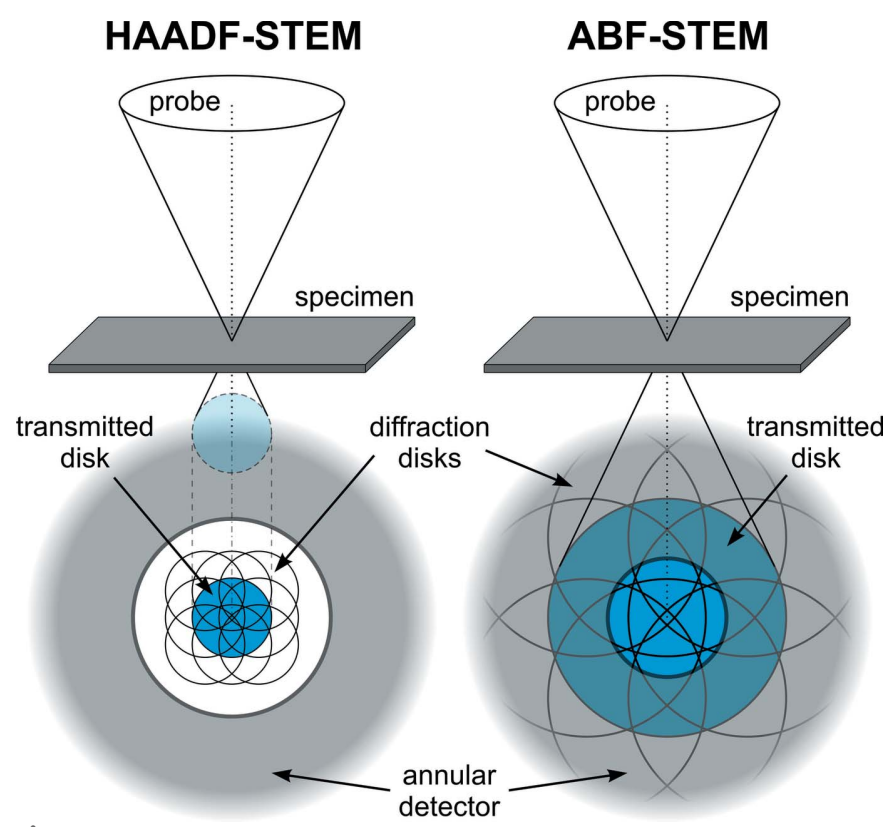

Figure 5

Schematic representation of the HAADF-STEM and ABF-STEM imaging techniques. Note the difference in the size of the transmitted disk (blue) with respect to the inner diameter of the annular detector (the outer diameter is not shown).
$Z$ number and/or large $Z$ number difference. The HAADF-STEM images of the $(\mathrm{Pb}, \mathrm{Bi})_{1-x} \mathrm{Fe}_{1+x^{-}}$ $\mathrm{O}_{3-y}$ compounds taken along the [010] zone axis are shown in Fig. 4 (bottom row). They reveal that the structures are composed of perovskite blocks, which appear as fragments with square patterns of bright dots $[(\mathrm{Pb}, \mathrm{Bi})$ atomic columns] with faint dots in between ( $\mathrm{Fe}-\mathrm{O}$ atomic columns). The blocks are separated by the CS planes, which interrupt the regular perovskite atomic arrangement and are visible as lines with short projected distances between the $(\mathrm{Pb}, \mathrm{Bi})$ columns as well as the $\mathrm{Fe}-$ $\mathrm{O}$ columns.

The steep almost quadratic dependence of the HAADF-STEM signal on the atomic number hinders the observation of the 'light' atomic columns, i.e. composed of the elements from the first and the second periods of the periodic table. However, knowing the cation positions with respect to the anionic sublattice is important, because it provides valuable information on the modulation of atomic displacements and interatomic distances. The position of the light scatterers can be directly visualized using annular bright-field (ABF) STEM imaging that, however, requires an aberration-corrected instrument. In this mode the inner collection angle of the detector is smaller than the probe convergence angle, so that the signal contains contributions of both coherently and incoherently scattered electrons (Fig. 5; Findlay et al., 2009, 2010). The ABF-STEM images demonstrate an absorptive-like contrast, where the regions of higher atomic potential look darker on a bright background. However, the dark spots attributed to the light and heavy atomic columns are different in origin. The atomic columns containing heavy elements scatter the electrons to high angles (into the HAADF range). The light atomic columns act more like electrostatic lenses, forward-focusing the electron beam, which then passes through the central hole of the detector. As a result the Z-dependence of the ABFSTEM signal cannot be strictly parameterized; yet for heavy elements it scales approximately as $Z^{1 / 3}$. The fact that the contrast for light and heavy atomic columns does not steeply depend on $Z$ allows very light atom columns to be visualized next to the columns of heavy cations (Batuk et al., 2011; Batuk, Batuk, Tsirlin et al., 2013; Ishikawa et al., 2011). Similar to HAADF-STEM, the contrast on the ABF-STEM images is quite robust to specimen thickness variations. The signal from the light atomic columns slowly vanishes with increasing thickness. However, this dependence is not crucial and does not significantly affect the interpretation of the image. Thus, a 
qualitative straightforward interpretation of the ABF-STEM images is possible, especially when the complementary HAADF-STEM images are available to ensure the correct assignment of the atomic columns. Taking into account that the modern aberration-corrected probe-forming optics allows routine registration of STEM images with sub-ångström resolution, the combination of the HAADF- and ABF-STEM techniques is a very powerful tool for retrieving structural information in direct space.

The interface-modulated structures, and the CS structures in particular, demonstrate smooth continuous behaviour of the atomic modulations only within the structure modules delimited by the interfaces. The changes at the interfaces are abrupt and are also accompanied by changes in the chemical composition, such as reducing the oxygen content. These abrupt changes have to be modelled in superspace using discontinuous modulation functions, dividing the $(3+1)$ dimensional structure into discrete atomic domains. Discontinuous changes in the occupancy factors (for example, switching between two types of atoms or between an atom and a vacancy) can be modelled with a step-like occupational function. This so-called crenel function is defined by two parameters, $\Delta$ and $x_{4}^{0}[\Delta$ is the width of the domain along the $x_{4}$ axis where the atom has the occupancy factor $g=1$ (outside the domain $g=0$ ), and $x_{4}^{0}$ is the centre of this domain (van der Lee et al., 1994; Petříček et al., 1995)]. The crenel occupancy modulation can be combined with linear atomic displacements resulting in a saw-tooth modulation defined as a function $\mathbf{U}=2 \mathbf{U}_{0}\left[\left(\bar{x}_{4}-x_{4}^{0}\right) / \Delta\right]$ on the crenel interval $\left(x_{4}^{0}-\Delta / 2\right.$; $\left.x_{4}^{0}+\Delta / 2\right)$, where $\mathbf{U}_{0}=\left(U_{0, x}, U_{0, y}, U_{0, z}\right)$ are the maximal displacement amplitudes (Petříček et al., 1990). The superspace model of the interface-modulated structure consists of a setup of step-like occupational functions defined for the constituting atomic positions. It should correctly reproduce the atomic arrangement within the structure modules confined by the interfaces as well as at the sequence of atomic layers at the interfaces. It also should establish unequivocally the relation between the components of the modulation vector and the chemical composition, i.e. the parameters of the occupational domains should be expressed as functions of the modulation vector components. Although such superspace models have already been applied to different CS structures (Michiue et al., 2005, 2006, 2007; Elcoro et al., 2012), so far there is no automated procedure which would allow derivation of such superspace models from either diffraction or imaging data. Such modelling becomes particularly challenging if only powder diffraction data are available. This is exactly the case where HAADF- and ABF-STEM imaging can provide a wealth of useful information.

With the example of the $(\mathrm{Pb}, \mathrm{Bi})_{1-x} \mathrm{Fe}_{1+x} \mathrm{O}_{3-y}$ CS structures, we will illustrate how the information on a discontinuous occupational modulation can be retrieved from the HAADF-STEM images in order to construct an occupational model of the structure in superspace. For these materials, HAADF-STEM images unambiguously reveal the sequence of atomic layers, and therefore provide just enough information for the construction of such a superspace model.
Although the CS planes can potentially adopt any possible $(h 0 l)_{\mathrm{p}}$ orientation, the atomic arrangement of the structure can be uniquely parameterized assuming that the CS planes consist of only two types of simple CS plane fragments, i.e. $(001)_{\mathrm{p}}$ and $(101)_{\mathrm{p}}$, see Fig. 6, and these fragments are uniformly distributed along the plane. We constructed the superspace model of the perovskite CS structures in the following steps:

(1) Identifying the sequences of atomic layers for the $(001)_{p}$ and $(101)_{\mathrm{p}}$ fragments.

(2) Creating two partial superspace models of the fictitious structures with periodic arrangement of the $(001)_{p}$ and $(101)_{p}$ CS planes, respectively. In each model we reproduced the atomic arrangement of the perovskite blocks and the CS planes with a set of step-like occupational modulation functions, whose parameters are related to the components of the modulation vector.

(3) Merging the partial models into a unified model of the structures with the $(h 0 l)_{\mathrm{p}}$ orientation of the CS planes employing the limiting conditions that $(h 0 l)_{\mathrm{p}} \rightarrow(001)_{\mathrm{p}}$ if $\alpha \rightarrow$ 0 and $(h 0 l)_{\mathrm{p}} \rightarrow(101)_{\mathrm{p}}$ if $\alpha \rightarrow \gamma$. It provides the link between the chemical composition of the series $(\mathrm{Pb}, \mathrm{Bi})_{1-{ }_{x}} \mathrm{Fe}_{1+\mathrm{x}} \mathrm{O}_{3-y}$ and the components of the modulation vector as $\mathrm{Pb}_{6 \gamma+2 \alpha} \mathrm{Bi}_{1-7 \gamma-\alpha} \mathrm{Fe}_{1+\gamma-\alpha} \mathrm{O}_{3-3 \gamma-\alpha} \quad$ (Abakumov et al., 2011).

The model utilizes only the components of the modulation vector as input parameters and adequately describes the atomic arrangement of any structure in the $(\mathrm{Pb}, \mathrm{Bi})_{1-x} \mathrm{Fe}_{1+x} \mathrm{O}_{3-y}$ family that exists over a broad range of Bi concentrations (from $\sim 10$ to 56 at. $\%$ in the $A$ positions).

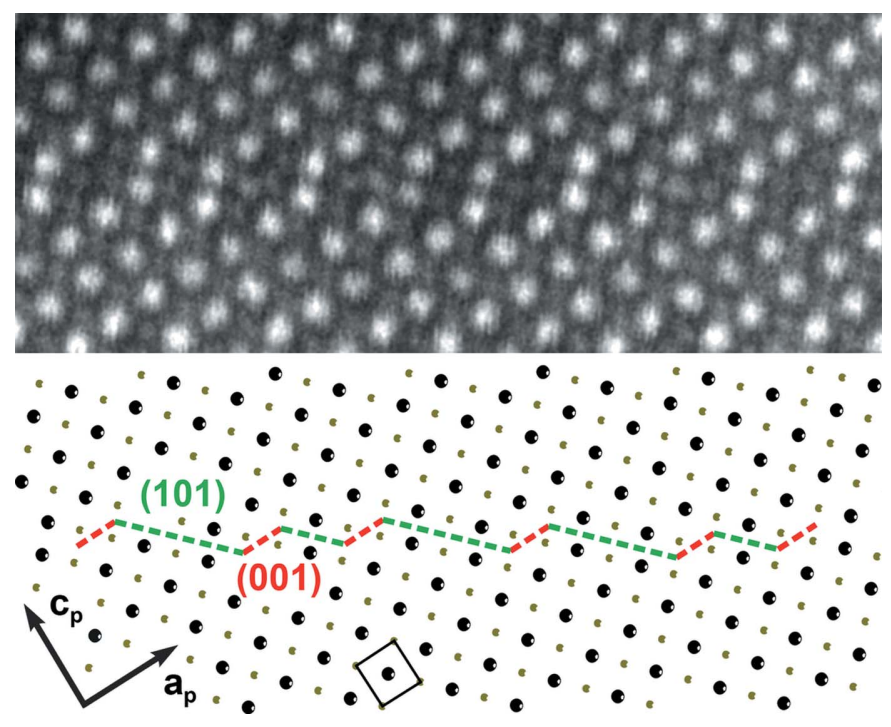

Figure 6

HAADF-STEM image of a CS plane in $(\mathrm{Pb}, \mathrm{Bi})_{1-{ }_{x}} \mathrm{Fe}_{1+x} \mathrm{O}_{3-y}$. Distribution of the atomic columns is reproduced below. The $(\mathrm{Pb}, \mathrm{Bi})$ columns are shown as black spheres (correspond to the brightest dots on the image), the Fe columns are shown as olive-green spheres (corresponding to the less bright dots on the image). The $(101)_{\mathrm{p}}$ and $(001)_{p}$ fragments of the CS planes are shown as green and red lines, respectively. Adapted with permission from Abakumov et al. (2011). Copyright (2011) American Chemical Society. 

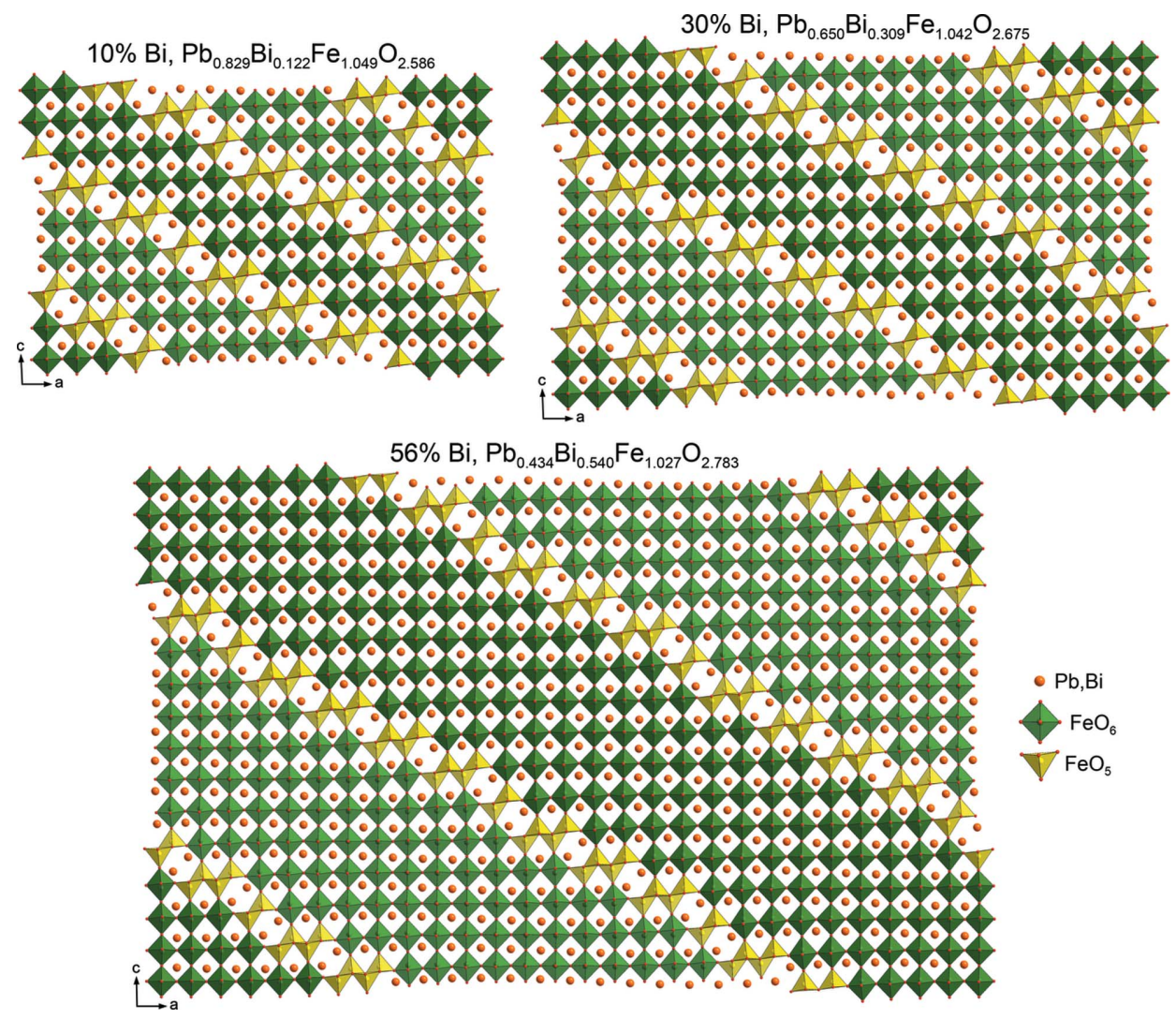

Figure 7

Representative fragments of three refined structures of the $(\mathrm{Pb}, \mathrm{Bi})_{1-x} \mathrm{Fe}_{1+x} \mathrm{O}_{3-y}$ family. $\mathrm{FeO}_{6}$ octahedra inside the perovskite blocks are shown in green, chains of double and quadruple $\mathrm{FeO}_{5}$ pyramids running along the $b$ direction are shown in yellow.

The model also provides a rather accurate chemical composition of the materials, which agrees with the EDX data. Slight systematic discrepancies are caused by the point oxygen vacancies present in the structure that are not accounted for in the superspace model (see the EDX results further in the

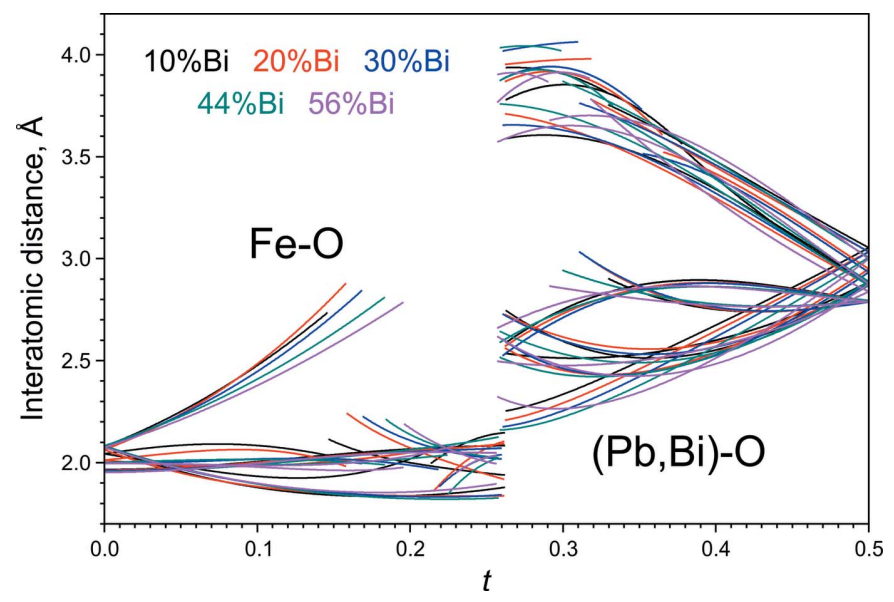

Figure 8

$t$-plot of the $\mathrm{Fe}-\mathrm{O}$ and $(\mathrm{Pb}, \mathrm{Bi})-\mathrm{O}$ interatomic distances in all the refined $(\mathrm{Pb}, \mathrm{Bi})_{1-x} \mathrm{Fe}_{1+x} \mathrm{O}_{3-y}$ structures. Plots for each individual structure can be found in Fig. S3 of the supporting information.

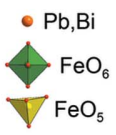

text). The validity of the constructed model has been confirmed using the Rietveld refinement against the NPD data of the $\mathrm{Pb}_{0.64} \mathrm{Bi}_{0.32} \mathrm{Fe}_{1.04} \mathrm{O}_{2.675}$ structure. The refinement demonstrated that the periodic arrangement of the CS planes imposes strong atomic displacement modulations, essential for stress relaxation at the interfaces.

Here we provide more extended analysis of the $(\mathrm{Pb}, \mathrm{Bi})_{1-x} \mathrm{Fe}_{1+x^{-}}$ $\mathrm{O}_{3-y}$ structure evolution materials upon variation of the chemical composition and associated changes in the thickness of the perovskite blocks. Using the constructed occupational superspace model, we refined the crystal structures of five compounds of the series with the Bi concentrations of $10,20,30,44$ and 56 at.\% in the $A$ cation sublattice. Details on the Rietveld refinement of these structures against NPD data acquired at $900 \mathrm{~K}$ (i.e. above the onset of the antiferromagnetic ordering) are provided in the supporting information. Representative fragments of three structures $\mathrm{Pb}_{0.829} \mathrm{Bi}_{0.122^{-}}$ $\mathrm{Fe}_{1.049} \mathrm{O}_{2.586}$ (10 at. \% $\left.\mathrm{Bi}\right), \mathrm{Pb}_{0.650^{-}}$ $\mathrm{Bi}_{0.309} \mathrm{Fe}_{1.042} \mathrm{O}_{2.675} \quad\left(30\right.$ at. $\%$ Bi) and $\mathrm{Pb}_{0.434} \mathrm{Bi}_{0.540^{-}}$ $\mathrm{Fe}_{1.027} \mathrm{O}_{2.783}(56$ at.\% $\mathrm{Bi}$ ) are shown in Fig. 7. All the five structures have very similar parameters of atomic displacive modulations for the $\mathrm{Fe},(\mathrm{Pb}, \mathrm{Bi})$ and three $\mathrm{O}$ atomic positions (Table S4 in the supporting information). The deformation patterns of the perovskite blocks in the structures can be illustrated with $t$-plots in Figs. 8 and 9. A $t$-plot represents the variation of a specific parameter plotted as a function of the internal coordinate $t, t=\bar{x}_{4}-\mathbf{q} \cdot \mathbf{r}$. The $t$-plot in Fig. 8 shows that the $\mathrm{Fe}-\mathrm{O}$ and $(\mathrm{Pb}, \mathrm{Bi})-\mathrm{O}$ distances in the structures change in a very similar fashion upon going from the centre of the perovskite blocks $[t=0$ for $\mathrm{Fe}$ and $t=0.5$ for $(\mathrm{Pb}, \mathrm{Bi})$ positions, respectively] towards the CS planes $(t \simeq 0.25)$. Fig. 9 shows the deformations of the $\mathrm{FeO}_{6}$ octahedra and $(\mathrm{Pb}, \mathrm{Bi}) \mathrm{O}_{12}$ cuboctahedra of the perovskite blocks parametrized with the octahedral and cuboctahedral distortion parameters, $\Delta_{\text {oct }}$ and $\Delta_{\text {cuboct }}$, respectively. They measure the distortion as a meansquare relative deviation of the $\mathrm{Fe}-\mathrm{O}$ and $(\mathrm{Pb}, \mathrm{Bi})-\mathrm{O}$ bond lengths from the average in $\mathrm{FeO}_{6}$ octahedra and $(\mathrm{Pb}, \mathrm{Bi}) \mathrm{O}_{12}$ cuboctahedra, so that $\Delta_{\text {oct }}=1 / 6 \sum_{i=1}^{6}\left[\left(l_{i}-\bar{l}\right) / \bar{l}\right]^{2}$ and $\Delta_{\text {cuboct }}=1 / 12 \sum_{i=1}^{12}\left[\left(l_{i}-\bar{l}\right) / \bar{l}\right]^{2}$, where $l_{i}$ are cation-oxygen distances [either $\mathrm{Fe}-\mathrm{O}$ or $(\mathrm{Pb}, \mathrm{Bi})-\mathrm{O}$ ] and $\bar{l}$ is a corresponding average value (Brown \& Shannon, 1973; Batuk, Batuk, Abakumov et al., 2013). In the (Pb,Bi $)_{1-x} \mathrm{Fe}_{1+x} \mathrm{O}_{3-y}$ materials, at the centre of the perovskite blocks, the under- 
lying perovskite matrix is practically undistorted $\left(\Delta_{\text {oct }}=4.0\right.$ $7.0 \times 10^{-4}$ and $\Delta_{\text {cuboct }}=6.0-9.0 \times 10^{-4}$ ), while going to the CS planes the structure acquires very strong polar distortions $\left(\Delta_{\text {oct }}=2.0-2.6 \times 10^{-2}\right.$ and $\left.\Delta_{\text {cuboct }}=3.0-4.1 \times 10^{-2}\right)$, but the directions of the atomic displacements on either side of the CS planes are the opposite resulting in the antipolar-type structure. The distortions on the periphery of the perovskite blocks are so strong that the corresponding $\Delta_{\text {oct }}$ and $\Delta_{\text {cuboct }}$ values are nearly as large as those for tetragonally distorted perovskites $\mathrm{PbVO}_{3}\left(\Delta_{\text {oct }}=4.0 \times 10^{-2}\right.$ and $\Delta_{\text {cuboct }}=$ $3.5 \times 10^{-2}$; Shpanchenko et al., 2004) and $\mathrm{BiCoO}_{3}\left(\Delta_{\text {oct }}=\right.$ $3.4 \times 10^{-2}$ and $\Delta_{\text {cuboct }}=5.2 \times 10^{-2}$; Oka et al., 2010), which demonstrate the largest polar distortions in perovskites. To verify the structure solution, we employed an ABF-STEM imaging that provides adequate visualization of the displacement modulations for light $\mathrm{O}$ atoms in the presence of heavy elements such as $\mathrm{Pb}, \mathrm{Bi}$ and Fe. Fig. 10 demonstrates excellent agreement of the simulated HAADF-STEM and ABF-STEM images, calculated using the refined structures, with the experimental ones for three compounds of the $(\mathrm{Pb}, \mathrm{Bi})_{1-x} \mathrm{Fe}_{1+x} \mathrm{O}_{3-y}$ series $\left(\mathrm{Pb}_{0.829} \mathrm{Bi}_{0.122} \mathrm{Fe}_{1.049} \mathrm{O}_{2.586}\right.$ with 10 at. $\% \mathrm{Bi}, \mathrm{Pb}_{0.650} \mathrm{Bi}_{0.309} \mathrm{Fe}_{1.042} \mathrm{O}_{2.675}$ with 30 at. $\% \mathrm{Bi}$ and $\mathrm{Pb}_{0.434} \mathrm{Bi}_{0.540} \mathrm{Fe}_{1.027} \mathrm{O}_{2.783}$ with 56 at.\% Bi). This comparison clearly shows that a combination of the HAADF-STEM and ABF-STEM imaging can pick up fine details of the occupational and displacive modulations.

\section{Chemical information}

Acquisition of STEM images can be combined with gathering spectroscopic information, thus enabling the analysis of chemically sensitive information with atomic resolution. Incident high-energy electrons interacting with an atom can transfer a small fraction of their energy to the inner-shell electrons of the atom, promoting them onto the unoccupied states. This energy loss is measured in electron energy loss spectroscopy (EELS). When the atom undergoes the transition back to the ground state by filling the hole with an electron from higher energy levels, it emits characteristic X-ray radiation, which is measured in energy-dispersive $\mathrm{X}$-ray (EDX) spectroscopy. While scanning the beam over the specimen, both EDX and EELS spectra can be recorded and used to identify the elements constituting the atomic columns (Okunishi et al., 2006; Bosman et al., 2007; Kimoto et al., 2007; Muller et al., 2008; Muller, 2009; D'Alfonso et al., 2010). Fine details of the electron energy loss near-edge structure (ELNES) carry information on the unoccupied local density of states. Mapping these details with high spatial resolution provides the distribution of the oxidation states and coordination numbers of transition metal cations at the atomic scale (Turner, Verbeeck et al., 2012; Tan et al., 2013).

Elemental mapping becomes particularly useful for structures where partial or complete ordering is expected of two elements that cannot be reliably discriminated either from Xray or electron diffraction (because of their close atomic numbers) or from neutron diffraction (because of close scattering lengths). In the $\mathrm{CS}$ structures these are $\mathrm{Pb}^{2+}$ and $\mathrm{Bi}^{3+}$ (with atomic numbers 82 and 83 , and coherent scattering lengths 9.405 and $8.532 \mathrm{fm}$, respectively). Chemically these cations are very similar: their ionic radii are very close and they both have a stereochemically active lone electron pair. One could expect a uniform distribution of these elements in the perovskite blocks of the CS structures. However, results of the atomic resolution EDX analysis of the $\mathrm{Pb}_{0.529} \mathrm{Bi}_{0.438} \mathrm{Fe}_{1.034} \mathrm{O}_{2.736}$ (44 at.\% $\mathrm{Bi}$ ) material reveal that the $\mathrm{Pb}$ and $\mathrm{Bi}$ distribution in the structure is not random (Fig. 11). In accordance with the requirement of electroneutrality, the $A$ positions at the CS planes are predominantly occupied by the $\mathrm{Pb}^{2+}$ cations. The $A$ positions in the perovskite blocks are jointly occupied by the $\mathrm{Pb}^{2+}$ and $\mathrm{Bi}^{3+}$ cations. However, contrary to the expected uniform distribution, the $\mathrm{Bi}^{3+}$ cations are concentrated on the periphery of the perovskite blocks and $\mathrm{Pb}^{2+}$ cations - at the centre. Such a distribution might indicate the presence of point $\mathrm{O}$ vacancies in the perovskite blocks favouring the occupation of the $A$ positions by the lower charge $\mathrm{Pb}^{2+}$ cations. The existence of this kind of defect in the CS structures has been put forward based on results of the Mössbauer spectroscopy on a $\mathrm{Pb}_{0.792} \mathrm{Sr}_{0.168} \mathrm{Fe}_{1.040} \mathrm{O}_{2.529}$ structure (Batuk, Batuk, Abakumov et al., 2013). Besides, the presence of the point $O$ vacancies explains slight yet systematic discrepancies in the experimentally determined composition of the materials and the composition established by the occupational superspace model.
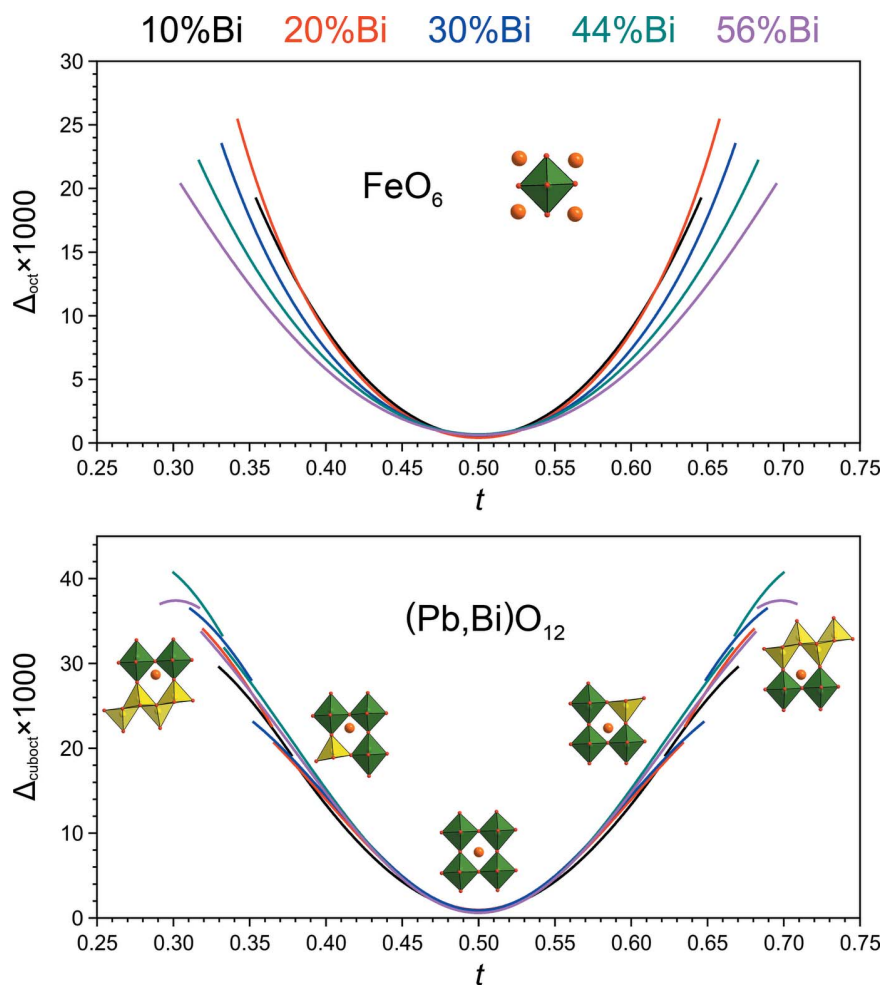

Figure 9

$t$-plot of the octahedral (upper panel) and cuboctahedral (bottom panel) distortion parameters for the refined $(\mathrm{Pb}, \mathrm{Bi})_{1-x} \mathrm{Fe}_{1+x} \mathrm{O}_{3-y}$ structures. Evolution of the $(\mathrm{Pb}, \mathrm{Bi})$ coordination environment in the perovskite blocks on going from the centre to the CS planes is schematically illustrated with the corresponding structural fragments. 
Mapping the coordination number of the transition metal cations provides information on the oxygen sublattice, which is complementary to the information extracted from the ABF-
STEM images (Batuk et al., 2014). The EELS spectra of the $\mathrm{Pb}_{2} \mathrm{Sr}_{2} \mathrm{Bi}_{2} \mathrm{Fe}_{6} \mathrm{O}_{16}$ structure with the (101) $)_{\mathrm{p}} \mathrm{CS}$ planes (Fig. 12) indicate that the fine structure of the Fe $L_{2,3}$ edge (the ELNES
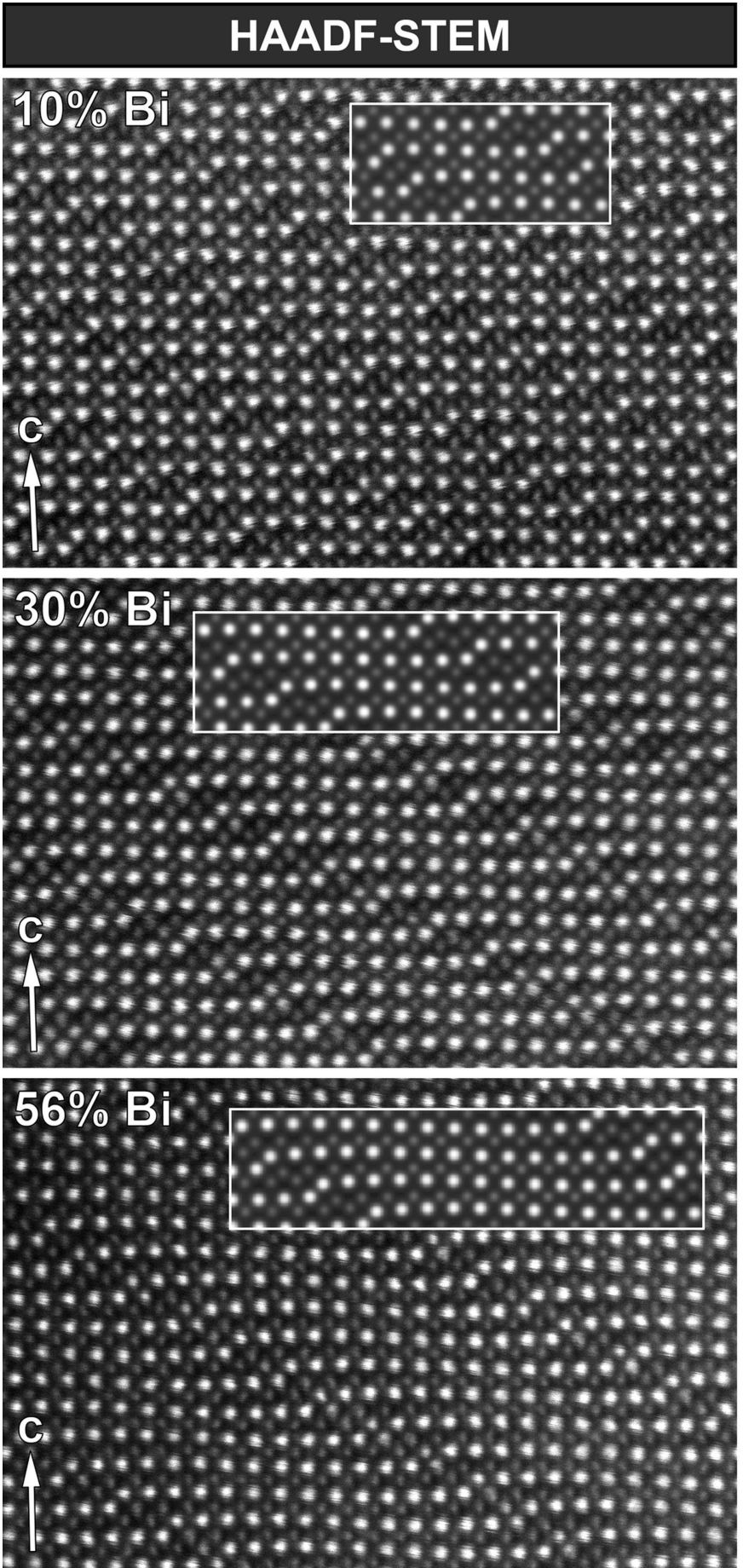

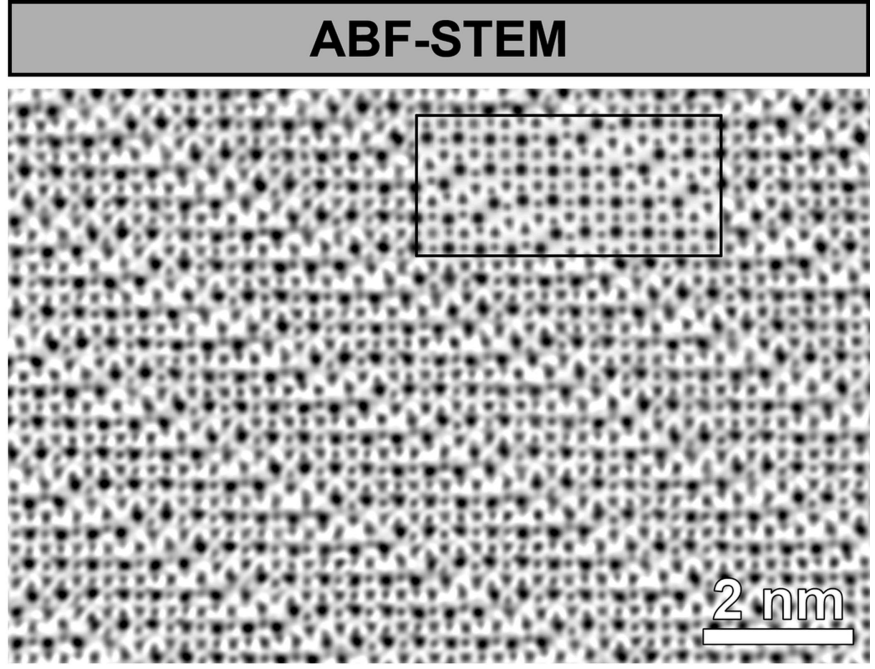

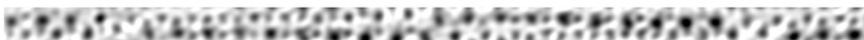

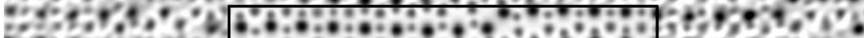

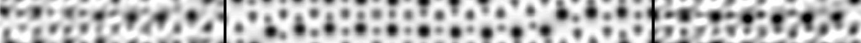

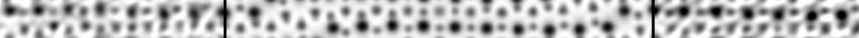
o

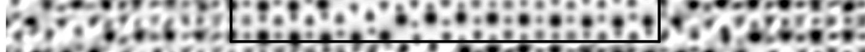

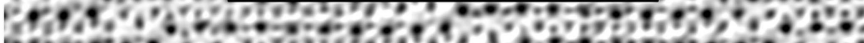

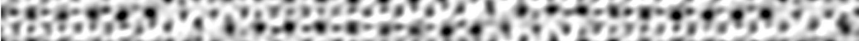

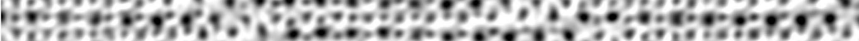

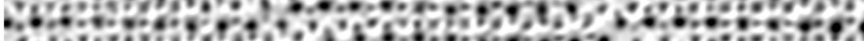

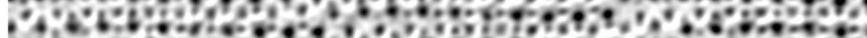

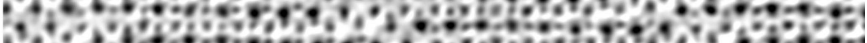

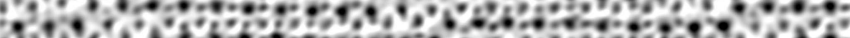
F,

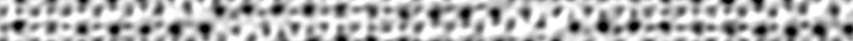
Go3.

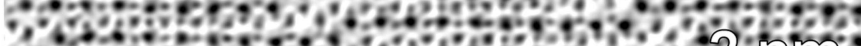

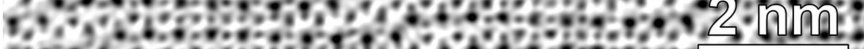

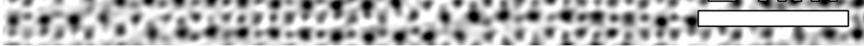

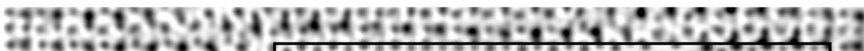

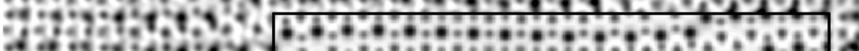

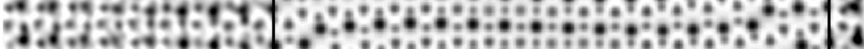

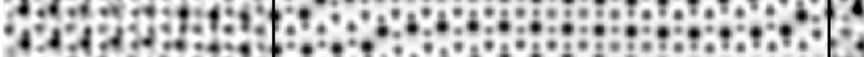

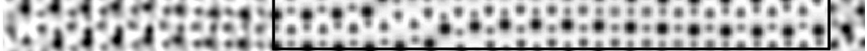

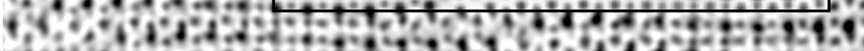

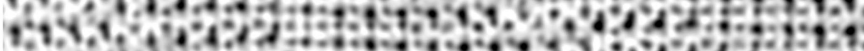

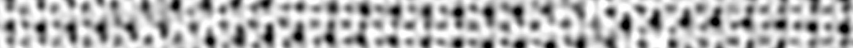

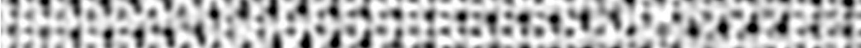

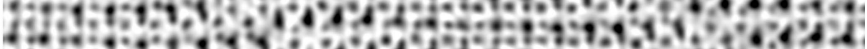

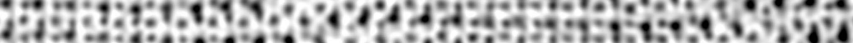

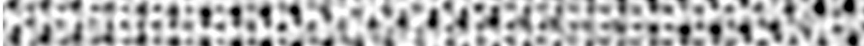

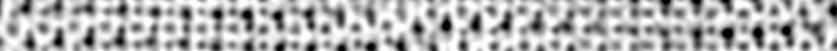

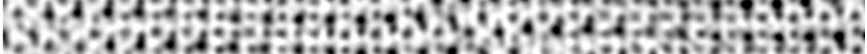

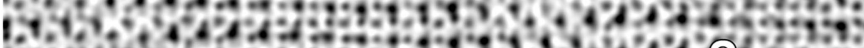

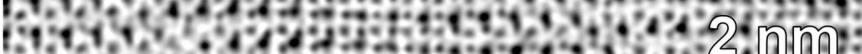

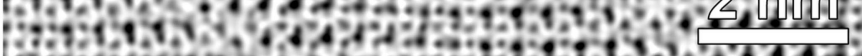

Figure 10

Complimentary HAADF-STEM and ABF-STEM images of the incommensurately modulated $(\mathrm{Pb}, \mathrm{Bi})_{1-x} \mathrm{Fe}_{1+x} \mathrm{O}_{3-y}$ perovskite CS structures with 10 at. $\% \mathrm{Bi}\left(\mathrm{Pb}_{0.829} \mathrm{Bi}_{0.122} \mathrm{Fe}_{1.049} \mathrm{O}_{2.586}\right), 30$ at. \% $\mathrm{Bi}\left(\mathrm{Pb}_{0.650} \mathrm{Bi}_{0.309} \mathrm{Fe}_{1.042} \mathrm{O}_{2.675}\right)$ and 56 at. $\% \mathrm{Bi}\left(\mathrm{Pb}_{0.434} \mathrm{Bi}_{0.540} \mathrm{Fe}_{1.027} \mathrm{O}_{2.783}\right)$. The HAADF-STEM images are shown as acquired, while the scanning noise on the ABF-STEM images has been removed by applying low-pass filtering. Bright and faint dots on the HAADF-STEM images correspond to the $(\mathrm{Pb}, \mathrm{Bi})$ and $\mathrm{Fe}-\mathrm{O}$ atomic columns in the structure, respectively. Note the presence of extra dots on the ABFSTEM images, which correspond to the pure O columns (see Fig. S5 of the supporting information for graphical representation). The insets demonstrate simulated STEM images calculated using crystallographic data for the refined structures. Note strong wave-like displacements of the $\mathrm{O}$ columns in all the structures. 
fine structure) measured from the CS planes is sufficiently different from that of the octahedral layers in the perovskite blocks. The $L_{3}$ peak of iron in octahedral coordination is split due to the crystal field splitting of the Fe $3 d$ energy level into $t_{2 g}$ and $e_{g}$ states. The splitting of the $L_{3}$ edge for the $\mathrm{Fe}^{3+}$ cations in square-pyramidal coordination at the CS planes is much less pronounced because of the lower symmetry of the coordination environment and the smaller crystal field splitting due to the lower coordination number (Turner, Egoavil et al., 2012).
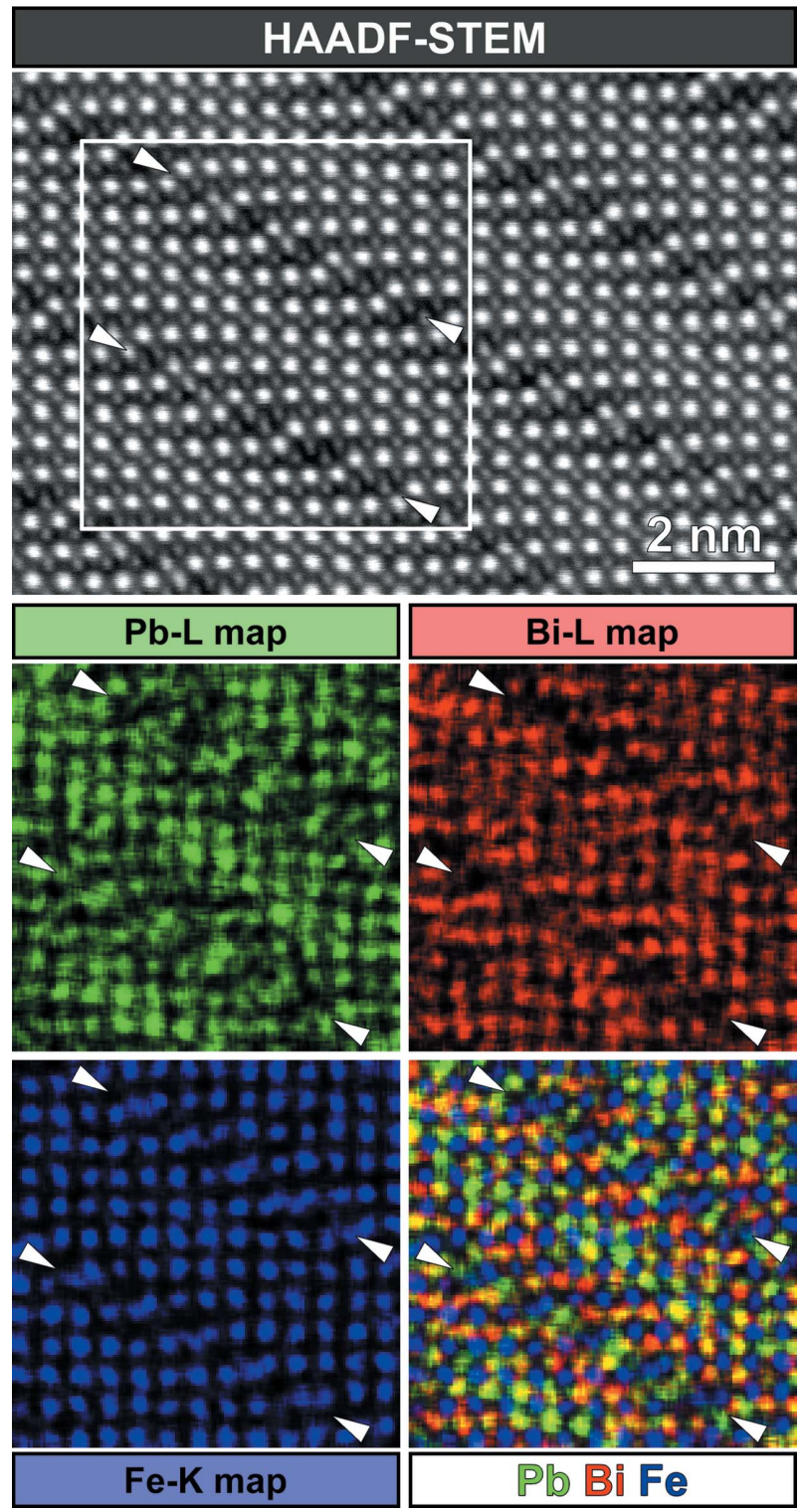

Figure 11

HAADF-STEM image of the $\mathrm{Pb}_{0.529} \mathrm{Bi}_{0.438} \mathrm{Fe}_{1.034} \mathrm{O}_{2.736}$ material with 44 at. $\%$ Bi together with the EDX-elemental maps for $\mathrm{Pb}-L, \mathrm{Bi}-L$ and $\mathrm{Fe}-K$ lines and the corresponding colour-mixed map. The arrowheads indicate the position and the orientation of the CS planes. Note that the $(\mathrm{Pb}, \mathrm{Bi})$ positions at the $\mathrm{CS}$ planes are depleted for $\mathrm{Bi}$. Inside the perovskite blocks, $\mathrm{Pb}$ is concentrated at the centre, while $\mathrm{Bi}$ is at the periphery.

\section{Concluding remarks}

We have demonstrated that a combination of advanced TEM techniques and powder diffraction is a powerful tool for the crystal structure solution of incommensurately modulated compounds. Using TEM, the crystallographic information can be collected at a very fine scale. Electron diffraction techniques allow three-dimensional reconstruction of the reciprocal space of the structures and can facilitate interpretation and indexing of powder diffraction data. With the development of
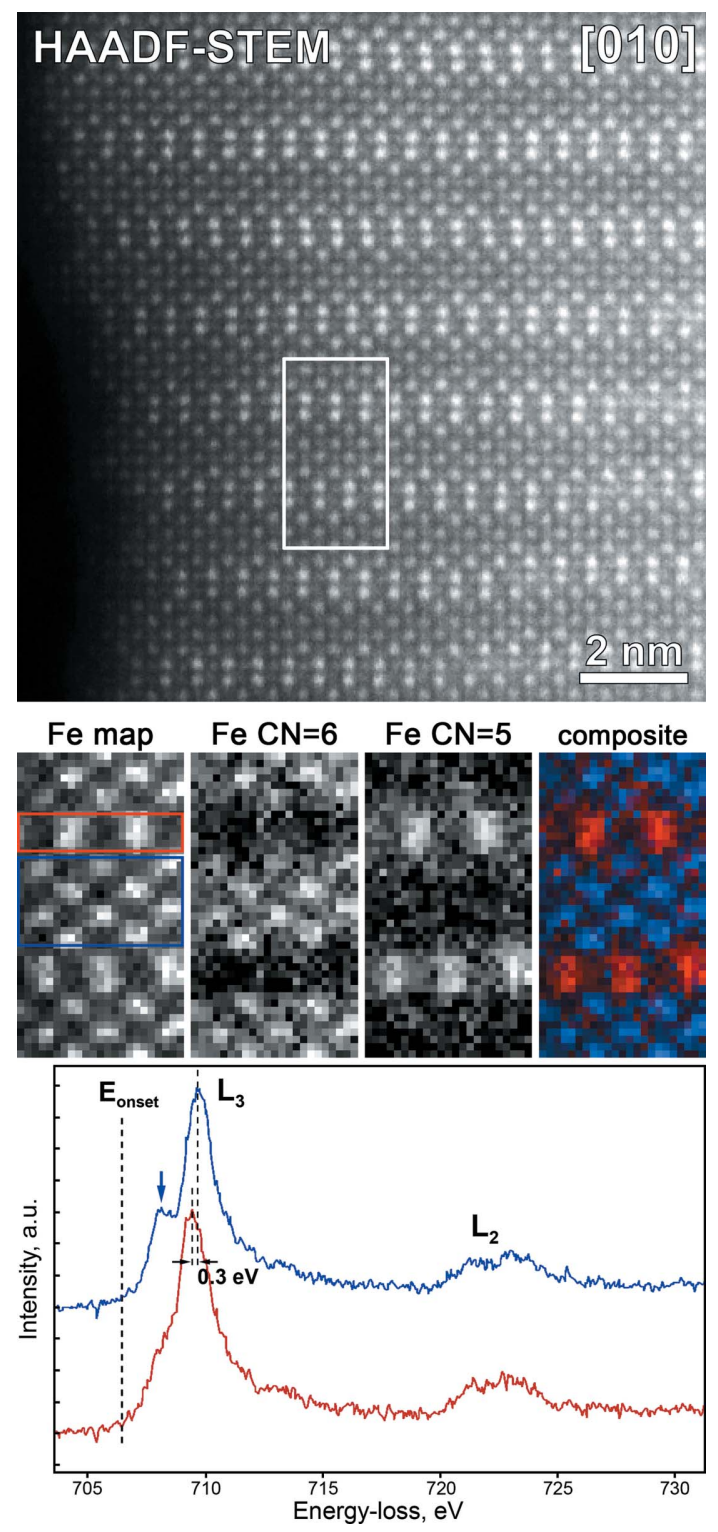

Figure 12

HAADF-STEM image of the $\mathrm{Pb}_{2} \mathrm{Sr}_{2} \mathrm{Bi}_{2} \mathrm{Fe}_{6} \mathrm{O}_{16}$ structure with the (101) $\mathrm{CS}$ planes (top panel) along with a Fe elemental map and maps of the $\mathrm{Fe}$ cations in octahedral (blue) and square pyramidal (red) coordination. The coordination maps are obtained by point-by-point decomposition of Fe $L_{2,3}$ edge spectra. Representative $\mathrm{Fe} L_{2,3}$ spectra for the $\mathrm{Fe}$ in octahedral and square pyramidal coordination are shown in the bottom panel. The spectra are integrated over large areas, which are marked with rectangles of the corresponding colour on the $\mathrm{Fe}$ map. The figure is adopted from Turner, Egoavil et al. (2012). 
new acquisition techniques, it is now possible to use electron diffraction data for the crystal structure solution, which can be subsequently employed as an input for the Rietveld refinement from powder diffraction data. Further progress in this direction might be expected in the development of full dynamical refinement procedure for aperiodic structures because it provides a possibility to account for the dynamical diffraction effects. Application of dynamical refinement from precession electron diffraction data to conventional periodic structures looks very promising (Palatinus et al., 2013).

HAADF- and ABF-STEM imaging with a sub-ångström resolution is capable of direct visualization of the structure, and therefore can be effectively used for extraction of the crystallographic information, structure model construction in superspace and validation of the crystal structure solution. Although a modulated structure can be directly visualized, the question remains how to convert images into numerical information, which can be directly employed in the Rietveld refinement from powder diffraction data. The observed atomic positions and their occupancies have to be expressed in superspace using the language of displacive and occupational modulation functions. As the parameters of the modulation functions are not directly observable quantities in the threedimensional direct space, a model-based image fitting procedure should be employed that links the experimental contrast to the parameters of the superspace model. A possibility to refine harmonic atomic displacement modulations for the fresnoite crystal structure from the HRTEM image has been demonstrated by Höche \& Neumann (2003). In this iterative procedure the optimization of the modulation parameters is based on the multislice image simulation of a commensurate supercell and minimization of the discrepancy between the calculated and experimental images. It might be possible that the proposed method would be even more effective if applied to incoherent HAADF-STEM images, because they can be roughly simulated as a convolution of the crystal projected potential with the Gaussian point-spread function of the electron probe (Van Aert et al., 2009). However, the correct conversion of HAADF intensities into occupancy factors might require a more elaborate treatment, based on the use of a statistical model-based method in combination with accurate frozen-phonon simulations accounting for the crystal thickness (Martinez et al., 2014).

The STEM-based spectroscopic methods provide valuable chemical information, which aids understanding building principles of the structure. HAADF-STEM imaging combined with the EELS or EDX compositional mapping provides a unique tool for investigating the distribution of elements with similar scattering power. It can resolve ambiguous situations where some crystallographic positions are simultaneously populated with more than two species (for example, two types of cations and cation vacancy) and varying the fractions of these species in a certain range does not affect the total scattering density at this position. Thus, the local crystallographic information acquired using the STEM-based methods in combination with the refinement from powder diffraction data can significantly improve the reliability of the crystal structure investigation.

\section{Related literature}

References cited in the supporting information include: Abakumov et al. (2007, 2013); Abakumov, Rossell et al. (2008); El Abed et al. (2003); Arakcheeva et al. (2008, 2012); Baldinozzi et al. (1998, 2000, 2002); Bereciartua et al. (2012); Bougerol-Chaillout et al. (2000); Claridge et al. (2009); Damay et al. (2008); Degtyareva et al. (2004); Deng et al. (2011); Dušek et al. (2010); Fredrickson et al. (2008); Fujihisa et al. (2005, 2007); Graetsch \& Brunelli (2005); Grebille et al. (2004); Hadermann et al. (2007); Hédoux et al. (1989); Hejny et al. (2005); Hejny \& McMahon (2003); Höche et al. (2003); Husband et al. (2012); Isobe et al. (2000, 2007); Jordan et al. (2003); Kawaguchi et al. (2013); Kenichi et al. (2003); Khasanova \& Antipov (1995); Larsson, Noren et al., (2007); Leineweber (2004, 2009); Leineweber et al. (2014); Li et al. (2005); Mandal et al. (2007); Michiue et al. (2004); Miyazaki et al. (2003, 2008); Miyazaki, Onoda, Edwards et al. (2002); Miyazaki, Onoda, Oku et al. (2002); Morozov, Mironov et al. (2006); Ohta et al. (1997); Onoda et al. (1999); Onoda \& Saeki (1993, 2004); Pauwels et al. (2006); Petrríček et al. (2001); Righi et al. (2010); Rivezzi \& Sciau (1998); Schefer et al. (2006); Sears (1992); Singh et al. (2013); Sławiński et al. (2009); van Smaalen et al. (2007); Tarakina et al. (2003); Wörle et al. (2006); Yamamoto et al. $(1990,1992)$.

\section{Acknowledgements}

The authors are grateful to the Laboratory for Neutron Scattering and Imaging of the Paul Scherrer Institut for granting the beam time at the HRPT diffractometer and to Dr Denis Sheptyakov for technical support during the experiments. The authors are thankful to Dr S. Turner for his help with the high-resolution STEM-EELS experiment and to Dr P. Oleynikov for collecting the rotation electron diffraction data. AMA appreciates the financial support from the Russian Science Foundation (grant 14-13-00680). Part of this work was supported by the Research Foundation Flanders under grant G.0392.11N.

\section{References}

Abakumov, A. M., Batuk, D., Hadermann, J., Rozova, M. G., Sheptyakov, D. V., Tsirlin, A. A., Niermann, D., Waschkowski, F., Hemberger, J., Van Tendeloo, G. \& Antipov, E. V. (2011). Chem. Mater. 23, 255-265.

Abakumov, A. M., Erni, R., Tsirlin, A. A., Rossell, M. D., Batuk, D., Nénert, G. \& Van Tendeloo, G. (2013). Chem. Mater. 25, 2670-2683.

Abakumov, A. M., Hadermann, J., Bals, S., Nikolaev, I. V., Antipov, E. V. \& Van Tendeloo, G. (2006). Angew. Chem. Int. Ed. 45, 66976700.

Abakumov, A. M., Hadermann, J., Van Tendeloo, G., Kovba, M. L., Skolis, Y. Y., Mudretsova, S. N., Antipov, E. V., Volkova, O. S., Vasiliev, A. N., Tristan, N., Klingeler, R. \& Büchner, B.(2007). Chem. Mater. 19, 1181-1189.

Abakumov, A. M., Hadermann, J., Van Tendeloo, G. \& Antipov, E. V. (2008). J. Am. Ceram. Soc. 91, 1807-1813. 
Abakumov, A. M., Mironov, A. V., Govorov, V. A., Lobanov, M. V., Rozova, M. G., Antipov, E. V., Lebedev, O. I. \& Van Tendeloo, G. (2003). Solid State Sci. 5, 1117-1125.

Abakumov, A. M., Morozov, V. A., Tsirlin, A. A., Verbeeck, J. \& Hadermann, J. (2014). Inorg. Chem. 53, 9407-9415.

Abakumov, A. M., Rossell, M. D., Gutnikova, O. Y., Drozhzhin, O. A., Leonova, L. S., Dobrovolsky, Y. A., Istomin, S. Y., Van Tendeloo, G. \& Antipov, E. V. (2008). Chem. Mater. 20, 44574467.

Altomare, A., Giacovazzo, C., Grazia, A., Moliterni, G. \& Rizzi, R. (2004). J. Res. Natl Inst. Stand. Technol. 109, 125-132.

Anderson, J. S. (1972). Surface and Defect Properties of Solids, edited by M. W. Roberts \& J. M. Thomas, pp. 1-53. Cambridge: Royal Society of Chemistry.

Anderson, J. S. \& Tilley, R. J. D. (1974). Surface and Defect Properties of Solids, edited by M. W. Roberts \& J. M. Thomas, pp. 1-56. Cambridge: Royal Society of Chemistry.

Arakcheeva, A. V., Logvinovich, D., Chapuis, G., Morozov, V. A., Eliseeva, S. V., Bünzli, J.-C. G. \& Pattison, P. (2012). Chem. Sci. 3, 384-390.

Arakcheeva, A. V., Pattison, P., Chapuis, G., Rossell, M., Filaretov, A., Morozov, V. \& Van Tendeloo, G. (2008). Acta Cryst. B64, 160171.

Baerlocher, C., McCusker, L. B. \& Palatinus, L. (2007). Z. Kristallogr. 222, 47-53.

Baldinozzi, G., Calvarin, G., Sciau, P., Grebille, D. \& Suard, E. (2000). Acta Cryst. B56, 570-576.

Baldinozzi, G., Goutenoire, F., Hervieu, M., Suard, E. \& Grebille, D. (1996). Acta Cryst. B52, 780-789.

Baldinozzi, G., Grebille, D., Sciau, P., Kiat, J.-M., Moret, J. \& Bérar, J.F. (1998). J. Phys. Condens. Matter, 10, 6461-6472.

Baldinozzi, G., Raulot, J.-M. \& Petrríček, V. (2002). MRS Proc. 755, 16.

Batuk, D., Batuk, M., Abakumov, A. M., Tsirlin, A. A., McCammon, C., Dubrovinsky, L. \& Hadermann, J. (2013). Inorg. Chem. 52, 10009-10020.

Batuk, M., Batuk, D., Tsirlin, A. A., Rozova, M. G., Antipov, E. V., Hadermann, J. \& Van Tendeloo, G. (2013). Inorg. Chem. 52, 22082218.

Batuk, D., Hadermann, J., Abakumov, A. M., Vranken, T., Hardy, A., Van Bael, M. \& Van Tendeloo, G. (2011). Inorg. Chem. 50, 49784986.

Batuk, M., Turner, S., Abakumov, A. M., Batuk, D., Hadermann, J. \& Van Tendeloo, G. (2014). Inorg. Chem. 53, 2171-2180.

Bereciartua, P. J., Zuñiga, F. J., Perez-Mato, J. M., Petříček, V., Vila, E., Castro, A., Rodríguez-Carvajal, J. \& Doyle, S. (2012). Acta Cryst. B68, 323-340.

Bird, D. M. \& Withers, R. L. (1986). J. Phys. Solid State Phys. 19, 3497-3505.

Bolotina, N. B. (2007). Crystallogr. Rep. 52, 647-658.

Bosman, M., Keast, V., García-Muñoz, J., D’Alfonso, A. J., Findlay, S. D. \& Allen, L. J. (2007). Phys. Rev. Lett. 99, 086102.

Bougerol-Chaillout, C., Bordet, P., Bérar, J. F., Darie, C. \& Pachot, S. (2000). Physica $C, \mathbf{3 4 1 - 3 4 8 , ~ 4 7 9 - 4 8 0 . ~}$

Bougerol, C., Gorius, M. \& Grey, I. (2002). J. Solid State Chem. 169, 131-138.

Boulahya, K., Ruiz-González, L., Parras, M., González-Calbet, J. M., Nickolsky, M. S. \& Nicolopoulos, S. (2007). Ultramicroscopy, 107, 445-452.

Boullay, P., Grebille, D., Hervieu, M., Raveau, B. \& Suard, E. (1999). J. Solid State Chem. 147, 450-463.

Boullay, P., Hervieu, M., Nguyen, N. \& Raveau, B. (1999). J. Solid State Chem. 147, 45-57.

Boullay, P., Palatinus, L. \& Barrier, N. (2013). Inorg. Chem. 52, $6127-$ 6135.

Boullay, P., Ténèze, N., Trolliard, G., Mercurio, D. \& Pérez-Mato, J. M. (2003). J. Solid State Chem. 174, 209-220.

Brown, I. D. \& Shannon, R. D. (1973). Acta Cryst. A29, 266-282.
Bursill, L. A. \& Hyde, B. G. (1972). J. Solid State Chem. 4, 430446.

Buseck, P. R. \& Cowley, J. M. (1983). Am. Mineral. 68, 18-40.

Claridge, J. B., Hughes, H., Bridges, C. A., Allix, M., Suchomel, M. R., Niu, H., Kuang, X., Rosseinsky, M. J., Bellido, N. \& Grebille, D. (2009). J. Am. Chem. Soc. 131, 14000-14017.

D'Alfonso, A. J., Freitag, B., Klenov, D. \& Allen, L. J. (2010). Phys. Rev. B, 81, 100101 .

Damay, F., Rodríguez-Carvajal, J., André, D., Dunstetter, F. \& Szwarc, H. (2008). Acta Cryst. B64, 589-595.

Degtyareva, O., McMahon, M. I. \& Nelmes, R. J. (2004). Phys. Rev. B, 70, 184119

Deng, G., Pomjakushin, V., Petricek, V., Pomjakushina, E., Kenzelmann, M. \& Conder, K. (2011). Phys. Rev. B, 84, 144111.

Dušek, M., Petř́iček, V. \& Palatinus, L. (2010). J. Phys. Conf. Ser. 226, 012014.

Dušek, M., Petříček, V., Wunschel, M., Dinnebier, R. E. \& van Smaalen, S. (2001). J. Appl. Cryst. 34, 398-404.

Eggeman, A. S. \& Midgley, P. A. (2012). Advances in Imaging and Electron Physics, edited by P. W. Hawkes, pp. 1-63. Amsterdam: Elsevier Inc.

El Abed, A., Gaudin, E., zur Loye, H.-C. \& Darriet, J. (2003). Solid State Sci. 5, 59-71.

Elcoro, L., Pérez, O., Perez-Mato, J. M. \& Petříček, V. (2012). Acta Cryst. B68, 341-355.

Fan, H. F. (1999). Microsc. Res. Tech. 46, 104-116.

Findlay, S. D., Shibata, N., Sawada, H., Okunishi, E., Kondo, Y. \& Ikuhara, Y. (2010). Ultramicroscopy, 110, 903-923.

Findlay, S. D., Shibata, N., Sawada, H., Okunishi, E., Kondo, Y., Yamamoto, T. \& Ikuhara, Y. (2009). Appl. Phys. Lett. 95, 191913.

Fischer, P., Frey, G., Koch, M., Könnecke, M., Pomjakushin, V., Schefer, J., Thut, R., Schlumpf, N., Bürge, R., Greuter, U., Bondt, S. \& Berruyer, E. (2000). Physica B, 276-278, 146-147.

Fredrickson, D. C., Lidin, S., Venturini, G., Malaman, B. \& Christensen, J. (2008). J. Am. Chem. Soc. 130, 8195-8214.

Fu, Z. Q., Huang, D. X., Li, F. H., Li, J. Q., Zhao, Z. X., Cheng, T. Z. \& Fan, H. F. (1994). Ultramicroscopy, 54, 229-236.

Fujihisa, H., Akahama, Y., Kawamura, H., Gotoh, Y., Yamawaki, H., Sakashita, M., Takeya, S. \& Honda, K. (2005). Phys. Rev. B, 72, 132103.

Fujihisa, H., Akahama, Y., Kawamura, H., Ohishi, Y., Gotoh, Y., Yamawaki, H., Sakashita, M., Takeya, S. \& Honda, K. (2007). Phys. Rev. Lett. 98, 175501.

Gemmi, M. \& Nicolopoulos, S. (2007). Ultramicroscopy, 107, 483-494.

Gillie, L. J., Hadermann, J., Pérez, O., Martin, C., Hervieu, M. \& Suard, E. (2004). J. Solid State Chem. 177, 3383-3391.

Gorelik, T. E., Stewart, A. A. \& Kolb, U. (2011). J. Microsc. 244, 325 331.

Graetsch, H. A. (2003). Z. Kristallogr. 218, 531-535.

Graetsch, H. A. (2007). Z. Kristallogr. 222, 226-233.

Graetsch, H. A. \& Brunelli, M. (2005). Z. Kristallogr. 220, 606-613.

Grebille, D., Lambert, S., Bourée, F. \& Petříček, V. (2004). J. Appl. Cryst. 37, 823-831.

Hadermann, J., Abakumov, A. M., Van Rompaey, S., Perkisas, T., Filinchuk, Y. \& Van Tendeloo, G. (2012). Chem. Mater. 24, 34013405.

Hadermann, J., Pérez, O., Creon, N., Michel, C. \& Hervieu, M. (2007). J. Mater. Chem. 17, 2344-2350.

Hao, Q., Liu, Y. \& Fan, H. (1987). Acta Cryst. A43, 820-824.

Hartel, P., Rose, H. \& Dinges, C. (1996). Ultramicroscopy, 63, 93-114.

Hédoux, A., Grebille, D. \& Garnier, P. (1989). Phys. Rev. B, 40, 10653-10656.

Hejny, C., Lundegaard, L. F., Falconi, S., McMahon, M. I. \& Hanfland, M. (2005). Phys. Rev. B, 71, 020101.

Hejny, C. \& McMahon, M. I. (2003). Phys. Rev. Lett. 91, 215502.

Höche, T., Esmaeilzadeh, S., Uecker, R., Lidin, S. \& Neumann, W. (2003). Acta Cryst. B59, 209-216.

Höche, T. \& Neumann, W. (2003). Ultramicroscopy, 96, 181-190. 
Husband, R. J., Loa, I., Stinton, G. W., Evans, S. R., Ackland, G. J. \& McMahon, M. I. (2012). Phys. Rev. Lett. 109, 095503.

Ishikawa, R., Okunishi, E., Sawada, H., Kondo, Y., Hosokawa, F. \& Abe, E. (2011). Nat. Mater. 10, 278-281.

Isobe, M., Onoda, M., Ohta, T., Izumi, F., Kimoto, K., TakayamaMuromachi, E., Hewat, A. W. \& Ohoyama, K. (2000). Phys. Rev. B, 62, 11667-11676.

Isobe, M., Onoda, M., Shizuya, M., Tanaka, M. \& TakayamaMuromachi, E. (2007). J. Am. Chem. Soc. 129, 14585-14596.

Isobe, M., Yoshida, H., Muromachi, E. T. \& Ohoyama, K. (2010). Sci. Technol. Adv. Mater. 11, 065004.

Izaola, Z., González, S., Elcoro, L., Perez-Mato, J. M., Madariaga, G. \& García, A. (2007). Acta Cryst. B63, 693-702.

Janner, A. \& Janssen, T. (1977). Phys. Rev. B, 15, 643-658.

Janssen, T., Chapuis, G. \& Boissieu, M. (2007). Aperiodic Crystals: From Modulated Phases to Quasicrystals. Oxford University Press. Janssen, T. \& Janner, A. (2014). Acta Cryst. B70, 617-651.

Janssen, T., Janner, A., Looijenga-Vos, A. \& de Wolff, P. M. (2006). International Tables for Crystallography, edited by E. Prince, pp. 907-955. Heidelberg: Springer.

Jordan, N. A., Battle, P. D., van Smaalen, S. \& Wunschel, M. (2003). Chem. Mater. 15, 4262-4267.

Kawaguchi, S., Ishibashi, H., Tsuji, N., Kim, J., Kato, K., Takata, M. \& Kubota, Y. (2013). J. Phys. Soc. Jpn, 82, 064603.

Kenichi, T., Kyoko, S., Hiroshi, F. \& Mitsuko, O. (2003). Nature, 423, 971-974.

Khasanova, N. R. \& Antipov, E. V. (1995). Physica C, 246, 241252.

Kimoto, K., Asaka, T., Nagai, T., Saito, M., Matsui, Y. \& Ishizuka, K. (2007). Nature, 450, 702-704.

Klein, H. (2013). Z. Kristallogr. 228, 35-42.

Koch, C. (2002). PhD thesis, Arizona State University, USA.

Kolb, U., Mugnaioli, E. \& Gorelik, T. E. (2011). Cryst. Res. Technol. 46, 542-554.

Larsson, A.-K., García-García, F. J. \& Withers, R. L. (2007). J. Solid State Chem. 180, 1093-1102.

Larsson, A.-K., Noren, L., Withers, R. L. \& Rundlöf, H. (2007). J. Solid State Chem. 180, 2723-2733.

Le Bail, A., Cranswick, L. M. D., Adil, K., Altomare, A., Avdeev, M., Cerny, R., Cuocci, C., Giacovazzo, C., Halasz, I., Lapidus, S. H., Louwen, J. N., Moliterni, A., Palatinus, L., Rizzi, R., Schilder, E. C., Stephens, P. W., Stone, K. H. \& van Mechelen, J. (2009). Powder Diffr. 24, 254-262.

Leineweber, A. (2004). J. Solid State Chem. 177, 1197-1212.

Leineweber, A. (2009). J. Solid State Chem. 182, 1846-1855.

Leineweber, A., Berger, T. G., Udyansky, A., Bugaev, V. N. \& Duppel, V. (2014). Z. Kristallogr. 229, 353-367.

Li, F. H. (1997). Mater. Chem. Phys. 47, 118-125.

Li, F. H. (2010). Phys. Status Solidi A, 207, 2639-2665.

Li, Z., Sun, J., You, L., Jiao, H., Li, G., Jing, X., Liao, F. \& Lin, J. (2005). Chem. Mater. 17, 2186-2192.

Liu, J., Li, F. H., Wan, Z. H., Fan, H. F., Wu, X. J., Tamura, T. \& Tanabe, K. (1998). Mater. Trans. JIM, 39, 920-926.

Magnéli, A., Hofman-Bang, N. \& Gjertsen, P. (1948). Acta Chem. Scand. 2, 501-517.

Mandal, T. K., Abakumov, A. M., Hadermann, J., Van Tendeloo, G., Croft, M. \& Greenblatt, M. (2007). Chem. Mater. 19, 6158-6167.

Martinez, G. T., Rosenauer, A., De Backer, A., Verbeeck, J. \& Van Aert, S. (2014). Ultramicroscopy, 137, 12-19.

Matsui, Y., Maeda, H., Tanaka, Y. \& Horiuchi, S. (1988). Jpn. J. Appl. Phys. 27, L361-L364.

McCusker, L. B. \& Baerlocher, C. (2009). Chem. Commun. pp. 14391451.

McCusker, L. B. \& Baerlocher, C. (2013). Z. Kristollgr. 228, 1-10.

Michiue, Y., Onoda, M., Brown, F. \& Kimizuka, N. (2004). J. Solid State Chem. 177, 2644-2648.

Michiue, Y., Yamamoto, A., Onoda, M., Sato, A., Akashi, T., Yamane, H. \& Goto, T. (2005). Acta Cryst. B61, 145-153.
Michiue, Y., Yamamoto, A. \& Tanaka, M. (2006). Acta Cryst. B62, 737-744.

Michiue, Y., Yamamoto, A. \& Tanaka, M. (2007). Philos. Mag. 87, 2655-2661.

Milne, S. J., Gard, J. A. \& West, A. R. (1985). Mater. Res. Bull. 20, 557-563.

Miyazaki, Y., Igarashi, D., Hayashi, K., Kajitani, T. \& Yubuta, K. (2008). Phys. Rev. B, 78, 214104.

Miyazaki, Y., Miura, T., Onoda, M., Uchida, M., Ishii, Y., Ono, Y., Morii, Y. \& Kajitani, T. (2003). Jpn. J. Appl. Phys. 42, 7467-7473.

Miyazaki, Y., Onoda, M., Edwards, P. P., Shamoto, S. \& Kajitani, T. (2002). J. Solid State Chem. 163, 540-545.

Miyazaki, Y., Onoda, M., Oku, T., Kikuchi, M., Ishii, Y., Ono, Y., Morii, Y. \& Kajitani, T. (2002). J. Phys. Soc. Jpn. 71, 491-497.

Mo, Y. D., Cheng, T. Z., Fan, H. F., Li, J. Q., Sha, B. D., Zheng, C. D., Li, F. H. \& Zhao, Z. X. (1992). Supercond. Sci. Technol. 5, 6972 .

Morozov, V. A., Arakcheeva, A. V., Chapuis, G., Guiblin, N., Rossell, M. D. \& Van Tendeloo, G. (2006). Chem. Mater. 18, 4075-4082.

Morozov, V. A., Arakcheeva, A. V., Konovalova, V. V., Pattison, P., Chapuis, G., Lebedev, O. I., Fomichev, V. V. \& Van Tendeloo, G. (2010). J. Solid State Chem. 183, 408-418.

Morozov, V. A., Bertha, A., Meert, K. W., Van Rompaey, S., Batuk, D., Martinez, G. T., Van Aert, S., Smet, P. F., Raskina, M. V., Poelman, D., Abakumov, A. M. \& Hadermann, J. (2013). Chem. Mater. 25, 4387-4395.

Morozov, V. A., Mironov, A. V., Lazoryak, B. I., Khaikina, E. G., Basovich, O. M., Rossell, M. D. \& Van Tendeloo, G. (2006). J. Solid State Chem. 179, 1183-1191.

Muller, D. A. (2009). Nat. Mater. 8, 263-270.

Muller, D. A., Kourkoutis, L. F., Murfitt, M., Song, J. H., Hwang, H. Y., Silcox, J., Dellby, N. \& Krivanek, O. L. (2008). Science, 319, 10731076.

Nellist, P. D. (2011). Scanning Transmission Electron Microscopy, edited by S. J. Pennycook \& P. D. Nellist, pp. 91-115. New York: Springer New York.

Ohta, T., Izumi, F., Onoda, M., Isobe, M., Takayama-Muromachi, E. \& Hewat, A. W. (1997). J. Phys. Soc. Jpn. 66, 3107-3114.

Oka, K., Azuma, M., Chen, W., Yusa, H., Belik, A. A., TakayamaMuromachi, E., Mizumaki, M., Ishimatsu, N., Hiraoka, N., Tsujimoto, M., Tucker, M. G., Attfield, J. P. \& Shimakawa, Y. (2010). J. Am. Chem. Soc. 132, 9438-9443.

Okunishi, E., Sawada, H., Kondo, Y. \& Kersker, M. (2006). Microsc. Microanal. 12, 1150-1151.

Onoda, M., Ishii, M., Pattison, P., Shibata, K., Yamamoto, A. \& Chapuis, G. (1999). J. Solid State Chem. 146, 355-362.

Onoda, M. \& Saeki, M. (1993). Jpn. J. Appl. Phys. 32, 752-753.

Onoda, M. \& Saeki, M. (2004). Solid State Ionics, 172, 481-484.

Onoda, M., Saeki, M., Yamamoto, A. \& Kato, K. (1993). Acta Cryst. B49, 929-936.

Onoda, M., Yamamoto, A., Takayama-Muromachi, E. \& Takekawa, S. (1988). Jpn. J. Appl. Phys. 27, L833-L836.

Palatinus, L. (2004). Acta Cryst. A60, 604-610.

Palatinus, L., Jacob, D., Cuvillier, P., Klementová, M., Sinkler, W. \& Marks, L. D. (2013). Acta Cryst. A69, 171-188.

Palatinus, L., Klementová, M., Dřínek, V., Jarošová, M. \& Petř́iček, V. (2011). Inorg. Chem. 50, 3743-3751.

Pauwels, D., Weill, F., Tressaud, A. \& Demourgues, A. (2006). Chem. Mater. 18, 6121-6131.

Pecharsky, V. K. \& Zavalij, P. Y. (2009). Fundamentals of Powder Diffraction and Structural Characterization of Materials. New York: Springer.

Petříček, V., Dušek, M. \& Palatinus, L. (2014). Z. Kristallogr. 229, 345-352.

Petř́íček, V., Elcoro, L., Pérez-Mato, J. M., Darriet, J., Teneze, N. \& Mercurio, D. (2001). Ferroelectrics, 250, 31-34.

Petříček, V., Gao, Y., Lee, P. \& Coppens, P. (1990). Phys. Rev. B, 42, 387-392. 
Petříček, V., van der Lee, A. \& Evain, M. (1995). Acta Cryst. A51, 529-535.

Righi, L., Albertini, F., Calestani, G., Pareti, L., Paoluzi, A., Ritter, C., Algarabel, P. A., Morellon, L. \& Ricardo Ibarra, M. (2006). J. Solid State Chem. 179, 3525-3533.

Righi, L., Albertini, F., Pareti, L., Paoluzi, A. \& Calestani, G. (2007). Acta Mater. 55, 5237-5245.

Righi, L., Albertini, F., Villa, E., Paoluzi, A., Calestani, G., Chernenko, V. A., Besseghini, S., Ritter, C. \& Passaretti, F. (2008). Acta Mater. 56, 4529-4535.

Righi, L., Lázpita, P., Gutierrez, J., Barandiaran, J. M., Chernenko, V. A. \& Calestani, G. (2010). Scr. Mater. 62, 383-386.

Rivezzi, N. \& Sciau, P. (1998). J. Solid State Chem. 139, 332341.

Rusakov, D. A., Abakumov, A. M., Yamaura, K., Belik, A. A., Van Tendeloo, G. \& Takayama-Muromachi, E. (2011). Chem. Mater. 23, 285-292.

Schefer, J., Schaniel, D., Pomjakushin, V., Stuhr, U., Petříček, V., Woike, T., Wöhlecke, M. \& Imlau, M. (2006). Phys. Rev. B, 74, 134103.

Schmid, S. \& Fung, V. (2012). Aust. J. Chem. 65, 851-859.

Sears, V. F. (1992). Neutron News, 3, 26-37.

Sha, B. D., Fan, H. F. \& Li, F. H. (1993). Acta Cryst. A49, 877880.

Shpanchenko, R. V., Chernaya, V. V., Tsirlin, A., Chizhov, P. S., Sklovsky, D. E., Antipov, E. V., Khlybov, E. P., Pomjakushin, V., Balagurov, A. M., Medvedeva, J. E., Kaul, E. E. \& Geibel, C. (2004). Chem. Mater. 16, 3267-3273.

Singh, S., Nayak, J., Rai, A., Rajput, P., Hill, A. H., Barman, S. R. \& Pandey, D. (2013). J. Phys. Condens. Matter, 25, 212203.

Singh, S., Petříček, V., Rajput, P., Hill, A. H., Suard, E., Barman, S. R. \& Pandey, D. (2014). Phys. Rev. B, 90, 014109.

Sławiński, W., Przeniosło, R., Sosnowska, I., Bieringer, M., Margiolaki, I. \& Suard, E. (2009). Acta Cryst. B65, 535-542.

Steeds, J. W., Bird, D. M., Eaglesham, D. J., McKernan, S., Vincent, R. \& Withers, R. L. (1985). Ultramicroscopy, 18, 97-110.

Stokes, H. T., Campbell, B. J. \& van Smaalen, S. (2011). Acta Cryst. A67, 45-55.

Tan, H., Egoavil, R., Béché, A., Martinez, G. T., Van Aert, S., Verbeeck, J., Van Tendeloo, G., Rotella, H., Boullay, P., Pautrat, A. \& Prellier, W. (2013). Phys. Rev. B, 88, 155123.

Tarakina, N. V., Tyutyunnik, A. P., Zubkov, V. G., D'Yachkova, T. V., Zainulin, Y. G., Hannerz, H. \& Svensson, G. (2003). Solid State Sci. 5, 983-994.

Tilley, R. J. D. (1970). Mater. Res. Bull. 5, 813-823.

Tilley, R. J. D. (1995). Int. J. Refract. Met. Hard Mater. 13, 93-109.
Turner, S., Egoavil, R., Batuk, M., Abakumov, A. M., Hadermann, J., Verbeeck, J. \& Van Tendeloo, G. (2012). Appl. Phys. Lett. 101, 241910.

Turner, S., Verbeeck, J., Ramezanipour, F., Greedan, J. E., Van Tendeloo, G. \& Botton, G. A. (2012). Chem. Mater. 24, 1904-1909.

Van Aert, S., Verbeeck, J., Erni, R., Bals, S., Luysberg, M., Van Dyck, D. \& Van Tendeloo, G. (2009). Ultramicroscopy, 109, 1236-1244.

van der Lee, A., Evain, M., Monconduit, L., Brec, R., Rouxel, J. \& Petříček, V. (1994). Acta Cryst. B50, 119-128.

Van Dyck, D., Broddin, D., Mahy, J. \& Amelinckx, S. (1987). Phys. Status Solidi A, 103, 357-373.

Van Landuyt, J., De Ridder, R., Gevers, R. \& Amelinckx, S. (1970). Mater. Res. Bull. 5, 353-362.

Van Landuyt, J., Van Tendeloo, G. \& Amelinckx, S. (1985). Pure Appl. Chem. 57, 1373-1382.

van Smaalen, S. (2004). Z. Kristallogr. 219, 681-691.

van Smaalen, S. (2007). Incommensurate Crystallography. Oxford University Press.

van Smaalen, S., Dinnebier, R., Sofin, M. \& Jansen, M. (2007). Acta Cryst. B63, 17-25.

Van Tendeloo, G., Bals, S., Van Aert, S., Verbeeck, J. \& Van Dyck, D. (2012). Adv. Mater. 24, 5655-5675.

Van Tendeloo, G., Hadermann, J., Abakumov, A. M. \& Antipov, E. V. (2009). J. Mater. Chem. 19, 2660-2670.

Vincent, R. \& Midgley, P. A. (1994). Ultramicroscopy, 53, 271-282.

Wadsley, A. D. (1955). Rev. Pure Appl. Chem. 5, 165-193.

Wagner, T. \& Schönleber, A. (2009). Acta Cryst. B65, 249-268.

Williams, D. B. \& Carter, C. B. (2009). Transmission Electron Microscopy. New York: Springer.

Withers, R. L. (1989). Prog. Cryst. Growth Charact. Mater. 18, 139204.

Withers, R. L., Norén, L. \& Liu, Y. (2004). Z. Kristallogr. 219, 701710.

Wolff, P. M. de (1974). Acta Cryst. A30, 777-785.

Wolff, P. M. de (1977). Acta Cryst. A33, 493-497.

Wörle, M., Nesper, R. \& Chatterji, T. K. (2006). Z. Anorg. Allg. Chem. 632, 1737-1742.

Xiang, S.-B., Fan, H.-F., Wu, X.-J., Li, F.-H. \& Pan, Q. (1990). Acta Cryst. A46, 929-934.

Yamamoto, A. (1996). Acta Cryst. A52, 509-560.

Yamamoto, A., Onoda, M., Takayama-Muromachi, E., Izumi, F., Ishigaki, T. \& Asano, H. (1990). Phys. Rev. B, 42, 4228-4239.

Yamamoto, A., Takayama-Muromachi, E., Izumi, F., Ishigaki, T. \& Asano, H. (1992). Physica C, 201, 137-144.

Zhang, D., Oleynikov, P., Hovmöller, S. \& Zou, X. (2010). Z. Kristallogr. 225, 94-102. 